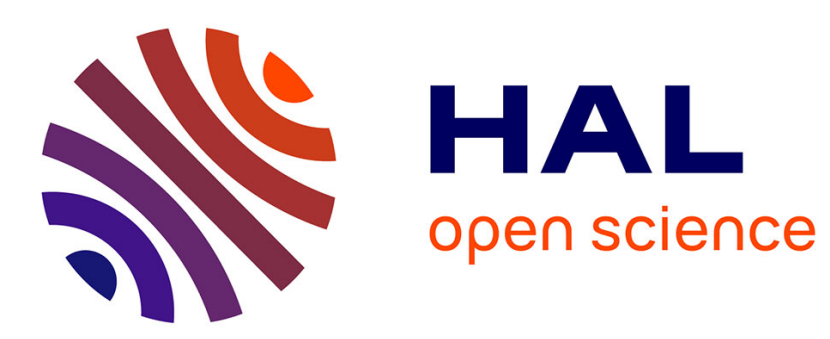

\title{
Global weak solutions to magnetic fluid flows with nonlinear Maxwell-Cattaneo heat transfer law
}

Fatah Aggoune, Kamel Hamdache, Djamila Hamroun

\section{To cite this version:}

Fatah Aggoune, Kamel Hamdache, Djamila Hamroun. Global weak solutions to magnetic fluid flows with nonlinear Maxwell-Cattaneo heat transfer law. 2014. hal-01023053

\section{HAL Id: hal-01023053 \\ https://hal.science/hal-01023053}

Submitted on 11 Jul 2014

HAL is a multi-disciplinary open access archive for the deposit and dissemination of scientific research documents, whether they are published or not. The documents may come from teaching and research institutions in France or abroad, or from public or private research centers.
L'archive ouverte pluridisciplinaire HAL, est destinée au dépôt et à la diffusion de documents scientifiques de niveau recherche, publiés ou non, émanant des établissements d'enseignement et de recherche français ou étrangers, des laboratoires publics ou privés. 


\title{
Global weak solutions to magnetic fluid flows with nonlinear Maxwell-Cattaneo heat transfer law
}

\author{
F. Aggoune*, K. Hamdache ${ }^{\dagger}$ and D. Hamroun ${ }^{\ddagger}$
}

\begin{abstract}
We discuss the equations describing the dynamic of the heat transfer in a magnetic fluid flow under the action of an applied magnetic field. Instead of the usual heat transfer equation we use a generalization given by the Maxwell-Cattaneo law which is a system satisfied by the temperature and the heat flux. We prove a global existence of weak solutions to the system having a finite energy.
\end{abstract}

Keywords : Navier-Stokes equations, Bloch-Torrey equation, magnetostatic equation, Maxwell-Cattaneo law, heat transfer, magnetic field, magnetization

AMS subject classifications: 76N10, 35Q35.

\section{Introduction}

\subsection{Statement of the model}

In this work, we study the heat transfer in a magnetic incompressible fluid flow under the action of an applied magnetic field. The temperature $\theta$ of the fluid is usually described by the linear heat transfer equation

$$
\partial_{t} \theta+U \cdot \nabla \theta=-\operatorname{div} Q
$$

related to the linear Fourier law

$$
Q=-\kappa \nabla \theta
$$

$Q$ being the heat flux and $U$ the fluid velocity. To ovoid the paradox of the instantaneous heat propagation inherent to the parabolic type equation, another model was offered in the pioneering work of Vernotte [23] and Cattaneo [6]. In this model, the Fourier law (2) is replaced by the heat-flux equation

$$
\tau \partial_{t} Q+Q=-\kappa \nabla \theta
$$

*Laboratoire AMNEDP, Faculté de Mathématiques. Université USTHB, Algérie (Email: aggoun@yahoot.fr)

${ }^{\dagger}$ Centre de Mathématiques Appliquées, CNRS UMR 7641 Ecole Polytechnique, 91128 Palaiseau cedex, France (Email: hamdache@cmapx.polytechnique.fr)

‡Laboratoire AMNEDP, Faculté de Mathématiques, Université USTHB, Algérie (Email: djamroun@yahoo.fr) 
where $\tau>0$ is the time relaxation parameter. For $\tau=0$, we recover equation (2). Combining the temperature equation and the heat-flux equation we see that $\theta$ satisfies an hyperbolic type equation. System (1)-(3) was generalized by Guyer and Krumhansl see [13] for example, by introducing a diffusion process in (3) so that the heat-flux equation becomes

$$
\tau\left(\partial_{t} Q-\gamma \Delta Q\right)=-Q-\kappa \nabla \theta
$$

where $\gamma>0$ is a diffusion coefficient. When the heat conductivity is enhanced by radiation effects see $[12,10,11]$, the linear Fourier law is replaced by a nonlinear one which writes aqs

$$
Q=-\nabla \mathcal{K}(\theta) .
$$

In [11], the model of heat transfer by the nonlinear Fourier law in an incompressible fluid flow has been discussed.

In this work we are dealing with the nonlinear Maxwell-Cattaneo law for heat transfer which is a generalization of the nonlinear Fourier law, more precisely we consider that the dynamic of the couple $(\theta, Q)$ is governed by the system

$$
\begin{aligned}
& \partial_{t} \theta+U \cdot \nabla \theta=-\operatorname{div} Q \\
& \tau\left(\partial_{t} Q-\gamma \Delta Q\right)=-\frac{\tau}{2} \operatorname{curl} U \times Q-Q-\nabla \mathcal{K}(\theta) .
\end{aligned}
$$

The monotone function $\mathcal{K}(\theta)$ discussed in this work is given by

$$
\mathcal{K}(\theta)=\kappa \theta+\alpha \theta^{3}
$$

where $\kappa>0$ and $\alpha>0$ are the heat conductivity coefficients and we refer the reader to $[19,20]$ for the introduction of the rotation term $\frac{1}{2} \operatorname{curl} U \times Q$. Notice that the power 3 used in the definition of the function $\mathcal{K}$ is less than the values indicated in [12].

The Maxwell-Cattaneo system (6) is coupled to the incompressible Navier-Stokes equations satisfied by the fluid velocity $U$ and the pressure $p$ as well as to the BlochTorrey equation satisfied by the magnetization field $M$ and the magnetostatic equation for the magnetic field $H$. Namely, we have

$$
\begin{aligned}
& \operatorname{div} U=0 \\
& \partial_{t} U+(U . \nabla) U-\eta \Delta U+\nabla p=-\rho(\theta) g+\mu_{0}(M . \nabla) H+\frac{\mu_{0}}{2} \operatorname{curl}(M \times H) \\
& \partial_{t} M+(U . \nabla) M-\sigma \Delta M+\frac{1}{\delta}\left(M-\chi_{0} H\right)=\frac{1}{2} \operatorname{curl} U \times H-\beta_{0} M \times(M \times H) \\
& \operatorname{div}(H+M)=F, \quad \operatorname{curl} H=0
\end{aligned}
$$

where the density $\rho(\theta)$ is given by the state of law

$$
\rho(\theta)=\rho_{0}\left(1-\beta\left(\theta-\theta^{0}\right)\right)
$$

where $\rho_{0}$ is the fluid density at the the temperature $\theta^{0}$ and $\beta$ is a physical coefficient. The function $g$ represents the force of gravity, $F$ is a function linked to the applied 
magnetic field and $\eta, \mu_{0}, \sigma, \delta, \chi_{0}, \beta_{0}>0$ are physical parameters.

When the magnetization $M$ is assumed to be in equilibrium state meaning it is parallel to the magnetic field $H$, the model in consideration is quite different from the one studied in this work. The magnetization law writes in general as

$$
M=\chi(\theta,|H|) H
$$

In that case, the Maxwell-Cattaneo system becomes

$$
\begin{gathered}
\partial_{t} \theta+U \cdot \nabla \theta+\mu_{0} \theta \frac{\partial M}{\partial \theta} \cdot(U \cdot \nabla) H=-\operatorname{div} Q+\eta \Phi(U) \\
\tau\left(\partial_{t} Q-\Delta Q+\frac{1}{2} \operatorname{curl} U \times Q\right)=-Q-\nabla \mathcal{K}(\theta)
\end{gathered}
$$

where $\mu_{0} \theta \frac{\partial M}{\partial \theta} \cdot(U \cdot \nabla) H$ is the thermal power and $\Phi(U)$ is the energy dissipation. The heat transfer problem in an incompressible fluid flow under the above Maxwell-Cattaneo law in a magnetic fluid is an open problem.

Let $D \subset \mathbb{R}^{3}$ be an open, bounded, regular and simply connected domain, with boundary $\Gamma$. For $T>0$ fixed, we set $D_{T}=(0, T) \times D$ and $\Gamma_{T}=(0, T) \times \Gamma$. The equations (6) and (8) are set on $D_{T}$ with the following initial and boundary conditions

$$
\begin{gathered}
U(0)=U_{0}, \operatorname{div} U_{0}=0, M(0)=M_{0}, \text { in } D \\
U=0, M \cdot \mathbf{n}=0, \operatorname{curl} M \times \mathbf{n}=0, H \cdot \mathbf{n}=0, \text { on } \Gamma_{T} \\
\theta(0)=\theta_{0}, Q(0)=Q_{0} \text { in } D \\
Q \times \mathbf{n}=0, \tau \gamma \operatorname{div} Q-\mathcal{K}(\theta)=0 \text { on } \Gamma_{T}
\end{gathered}
$$

where $\mathbf{n}$ represents the unit outward normal to the boundary $\Gamma$. Problem (6)-(8)-(12)(13) will be labeled problem $(\mathcal{P})$.

System (8) with the temperature equation (1) has been discussed in [1, 2]. The linear Maxwell-Cattaneo system (1)-(3) has been studied in [14, 15] in the case where the velocity $U$ is fixed.

\section{$1.2 \quad$ Notations and spaces}

For $1 \leq q \leq \infty$ and $s \in \mathbb{R}$, let $L^{q}(D)$ and $W^{s, q}(D)$ be the usual Lebesgue and Sobolev spaces of scalar functions. If $q=2, W^{s, 2}(D)$ is denoted by $H^{s}(D)$ and $\|\cdot\|$ and $(\cdot ; \cdot)$ denote the norm and the scalar product of the Hilbert space $L^{2}(D)$. For vector valued functions we use the notations $\mathbb{L}^{q}(D), \mathbb{W}^{s, q}(D), \mathbb{H}^{s}(D)$ and the notations of norm and the scalar product of $\mathbb{L}^{2}(D)$ are unchanged. If $\mathcal{V}$ is a Banach space we denote by $\langle\cdot ; \cdot\rangle_{\mathcal{V}^{\prime} \times \mathcal{V}}$ (or simply $\langle\cdot ; \cdot\rangle$ if no confusion arises) the duality product where $\mathcal{V}^{\prime}$ is the dual space of $\mathcal{V}$. If $V$ is an Hilbert space with scalar product $(\cdot ; \cdot)$, we set

$$
\mathcal{C}([0, T] ; V \text { weak })=\{u:[0, T] \rightarrow V ;(u(\cdot), v) \in \mathcal{C}([0, T]), \forall v \in V\} .
$$


Let $\mathcal{D}\left(D, \mathbb{R}^{3}\right)$ the set of functions $f: D \rightarrow \mathbb{R}^{3}$ which are infinitely differentiable with compact support in $D$ and $\mathbb{H}_{0}^{1}(D)$ its closure in $\mathbb{H}^{1}(D)$. Now, we introduce the functional spaces used in the theory of Navier-Stokes equations, see $[22,7]$ for example

$$
\begin{gathered}
\mathcal{D}_{s}(D)=\left\{v \in \mathcal{D}\left(D, \mathbb{R}^{3}\right) ; \operatorname{div} v=0 \text { in } D\right\} \\
\mathcal{U}=\text { closure of } \mathcal{D}_{s}(D) \text { in } \mathbb{H}^{1}(D), \mathcal{U}_{0}=\text { closure of } \mathcal{D}_{s}(D) \text { in } \mathbb{L}^{2}(D) .
\end{gathered}
$$

Then it is well known that

$$
\begin{gathered}
\mathcal{U}=\left\{v \in \mathbb{H}_{0}^{1}(D) ; \operatorname{div} v=0 \text { in } D\right\} \\
\mathcal{U}_{0}=\left\{v \in \mathbb{L}^{2}(D) ; \operatorname{div} v=0 \text { in } D, v \cdot \mathbf{n}=0 \text { on } \Gamma\right\}
\end{gathered}
$$

and identifying $\mathcal{U}_{0}$ with its dual, we get as usual the inclusions $\mathcal{U} \subset \mathcal{U}_{0} \subset \mathcal{U}^{\prime}$.

For the Bloch-Torrey equation satisfied by $M$ and the heat-flux equation satisfied by $Q$ we introduce the Hilbert spaces

$$
\begin{aligned}
& \mathbb{H}_{t}^{1}(D)=\left\{M \in \mathbb{H}^{1}(D) ; M \cdot \mathbf{n}=0 \text { on } \Gamma\right\} \\
& \mathbb{H}_{n}^{1}(D)=\left\{Q \in \mathbb{H}^{1}(D) ; Q \times \mathbf{n}=0 \text { on } \Gamma\right\}
\end{aligned}
$$

equipped with the norm of $\mathbb{H}^{1}(D)$. Then (see [7] for example) there exists $C>0$ such that for all $V$ in either $\mathbb{H}_{t}^{1}(D)$ or $\mathbb{H}_{n}^{1}(D)$ the following estimate holds

$$
\|\nabla V\| \leq C\left(\|V\|^{2}+\|\operatorname{curl} V\|^{2}+\|\operatorname{div} V\|^{2}\right)^{1 / 2}
$$

hence the norm of $\mathbb{H}^{1}(D)$ is equivalent to the norm $\left(\|V\|^{2}+\|\operatorname{curl} V\|^{2}+\|\operatorname{div} V\|^{2}\right)^{1 / 2}$ on the spaces $\mathbb{H}_{t}^{1}(D)$ and $\mathbb{H}_{n}^{1}(D)$. We recall the relation $-\Delta=\operatorname{curl}^{2}-\nabla$ div so that for regular vector fields $\Psi$ and $\Phi$ the following Green formula holds

$$
\begin{array}{r}
-\int_{D} \Delta \Psi \cdot \Phi d x=\int_{D} \operatorname{curl} \Psi \cdot \operatorname{curl} \Phi d x+\int_{D} \operatorname{div} \Psi \operatorname{div} \Phi d x \\
+\int_{\Gamma} \operatorname{curl} \Psi \cdot(\Phi \times \mathbf{n}) d \Gamma-\int_{\Gamma} \operatorname{div} \Psi(\Phi \cdot \mathbf{n}) d \Gamma .
\end{array}
$$

To deal with the magnetostatic equation, we set

$$
L_{\sharp}^{2}=\left\{\psi \in L^{2}(D) ; \int_{D} \psi(x) d x=0\right\} \text { and } H_{\sharp}^{1}=H^{1}(D) \cap L_{\sharp}^{2} .
$$

The Hilbert space $H_{\sharp}^{1}$ is equipped with the norm $\|\nabla \psi\|$ which is equivalent to the usual norm of $H^{1}(D)$ thanks to Poincaré-Wirtinger inequality : there exists $C>0$ such that for all $\psi \in \mathbb{H}_{\sharp}^{1}$ we have

$$
\|\psi\| \leq C\|\nabla \psi\|
$$

To end these notations, we point out that throughout this paper, $C>0$ indicates a generic constant depending only on some bounds of the physical data, which takes different values in different occurrences. The dependency of the constants $C>0$ with respect to a parameter $m$ is written as $C_{m}$.

Now, let us focus our attention on the magnetostatic equation to give some useful continuity results on the solution 


\subsection{The magnetostatic equation}

Let $M \in \mathbb{L}^{2}(D)$ and $F \in L_{\sharp}^{2}$, we consider the following problem

Find $\varphi \in H_{\sharp}^{1} ; \quad \forall \psi \in H_{\sharp}^{1}, \quad \int_{D}(\nabla \varphi+M) \cdot \nabla \psi d x=-\int_{D} F \psi d x$.

This problem admits a unique solution $\varphi$ in $H_{\sharp}^{1}$ and we have

$$
\int_{D} \nabla \varphi \cdot M d x=-\|\nabla \varphi\|^{2}-\int_{D} F \varphi d x
$$

then

$$
\|\nabla \varphi\| \leq(\|M\|+C\|F\|) .
$$

In particular the application

$$
\mathcal{H}:(M, F) \mapsto \varphi
$$

is continuous from $\mathbb{L}^{2}(D) \times L_{\sharp}^{2}$ to $H_{\sharp}^{1}$. Furthermore testing equation (17) with $\psi-$ $\int_{D} \psi d x, \psi \in H^{1}(D)$, we see that

$$
\int_{D}(\nabla \varphi+M) \cdot \nabla \psi d x=-\int_{D} F \psi d x, \quad \forall \psi \in H^{1}(D)
$$

and $H=\nabla \varphi$ solves the problem

$$
\left\{\begin{array}{l}
\operatorname{div}(H+M)=F, \quad \operatorname{curl} H=0 \text { in } D \\
(H+M) \cdot \mathbf{n}=0 \text { on } \Gamma .
\end{array}\right.
$$

Moreover using classical regularity results for elliptic problems, we conclude that if $F \in L_{\sharp}^{2}$ and $M \in \mathbb{H}_{t}^{1}(D)$, then $\varphi \in \mathbb{H}^{2}(D) \cap H_{\sharp}^{1}$ and we have

$$
\|\varphi\|_{H^{2}(D)} \leq C(\|\operatorname{div} M\|+\|F\|)
$$

Therefore $H=\nabla \varphi \in \mathbb{H}_{t}^{1}(D)$ and we have

$$
\|H\|_{H^{1}(D)} \leq C(\|\operatorname{div} M\|+\|F\|) .
$$

We can see that $\mathcal{H}$ is also continuous from $\mathbb{L}^{2}\left(D_{T}\right) \times L^{2}\left(0, T ; L_{\sharp}^{2}\right)$ to $L^{2}\left(0, T ; H_{\sharp}^{1}\right)$ and from $H^{1}\left(0, T ; \mathbb{L}^{2}(D)\right) \times H^{1}\left(0, T ; L_{\sharp}^{2}\right)$ to $H^{1}\left(0, T ; H_{\sharp}^{1}\right)$. Moreover for $F \in H^{1}\left(0, T ; L_{\sharp}^{2}\right)$ and $M \in H^{1}\left(0, T ; \mathbb{L}^{2}(D)\right)$, we have

$$
\int_{D}\left(\nabla\left(\partial_{t} \varphi\right)+\partial_{t} M\right) \cdot \nabla \psi d x=-\int_{D} \partial_{t} F \psi d x, \forall \psi \in H^{1}(D), \quad t \in(0, T) .
$$

\section{Main results}

Before stating our main result, let us give the formal energy estimates for problem $(\mathcal{P})$. 


\subsection{Energy estimates}

Let $(U, M, H, \theta, Q)$ be a regular solution to system $(\mathcal{P})$. We proceed as in $[1,2]$ to obtain, for $\theta$ fixed, the energy estimate satisfied by $(U, M, H)$. For $t \in[0, T]$, we set

$$
\begin{aligned}
& \mathcal{E}_{n s b t}(t)=\frac{1}{2}\|U(t)\|^{2}+\frac{\mu_{0}}{2}\left(\|M(t)\|^{2}+\|H(t)\|^{2}\right) \\
& \mathcal{E}_{n s b t, 0}=\frac{1}{2}\left\|U_{0}\right\|^{2}+\frac{\mu_{0}}{2}\left(\left\|M_{0}\right\|^{2}+\left\|H_{0}\right\|^{2}\right)
\end{aligned}
$$

where $H_{0}=\nabla \varphi_{0}$ and $\varphi_{0}$ is the unique solution of the following problem (see subsection $1.3)$

Find $\varphi_{0} \in H_{\sharp}^{1}$ such that

$$
\int_{D}\left(\nabla \varphi_{0}+M_{0}\right) \cdot \nabla \psi d x=-\int_{D} F(0) \psi d x, \quad \forall \psi \in H_{\sharp}^{1}
$$

and

$$
\begin{gathered}
\mathcal{F}_{n s b t}(t)=\eta\|\nabla U(t)\|^{2}+\mu_{0} \sigma\left(\|\operatorname{curl} M(t)\|^{2}+2\|\operatorname{div} M(t)\|^{2}\right)+\frac{\mu_{0}}{\delta}\|M(t)\|^{2}+ \\
\frac{\mu_{0}}{\delta}\left(1+2 \chi_{0}\right)\|H(t)\|^{2}+\beta_{0} \mu_{0}\|M(t) \times H(t)\|^{2} .
\end{gathered}
$$

Then we get the energy estimate

$$
\mathcal{E}_{n s b t}(t)+\int_{0}^{t} \mathcal{F}_{n s b t}(s) d s \leq \mathcal{E}_{n s b t, 0}+C \int_{0}^{t}\|\rho(\theta(s))\|^{2} d s+C \int_{0}^{t}\|G(s)\|^{2} d s
$$

for all $t \geq 0$ where

$$
G(t)=\|F(t)\|^{2}+\left\|\partial_{t} F(t)\right\|^{2} .
$$

Now we consider the Maxwell-Cattaneo system (6) satisfied by $(\theta, Q)$ for $U$ fixed. Let $\varpi$ the primitive function of $\mathcal{K}$ defined by

$$
\varpi(\theta)=\frac{\kappa}{2} \theta^{2}+\frac{\alpha}{4} \theta^{4} .
$$

Multiplying the temperature equation by $\mathcal{K}(\theta)$ and the heat-flux equation by $Q$ then integrating by parts and adding both results, we get the energy estimate associated with the Maxwell-Cattaneo system

$$
\mathcal{E}_{m c}(t)+\int_{0}^{t} \mathcal{F}_{m c}(s) d s \leq \mathcal{E}_{m c, 0}
$$

for all $t \geq 0$ with

$$
\begin{gathered}
\mathcal{E}_{m c}(t)=\int_{D} \varpi(\theta(t)) d x+\frac{\tau}{2}\|Q(t)\|^{2}, \quad \mathcal{E}_{m c, 0}=\int_{D} \varpi\left(\theta_{0}\right) d x+\frac{\tau}{2}\left\|Q_{0}\right\|^{2} \\
\mathcal{F}_{m c}(t)=\tau \gamma\left(\|\operatorname{curl} Q(t)\|^{2}+\|\operatorname{div} Q(t)\|^{2}\right)+\|Q(t)\|^{2} .
\end{gathered}
$$

The total energy $\mathcal{E}$ and the total dissipation energy $\mathcal{F}$ of the full problem $(\mathcal{P})$ are defined by

$$
\mathcal{E}(t)=\mathcal{E}_{n s b t}(t)+\mathcal{E}_{m c}(t), \quad \mathcal{F}(t)=\mathcal{F}_{n s b t}(t)+\mathcal{F}_{m c}(t)
$$

and it holds

$$
\mathcal{E}(t)+\int_{0}^{t} \mathcal{F}(s) d s \leq \mathcal{E}_{0}+C \int_{0}^{t}\|\rho(\theta(s))\|^{2} d s+C \int_{0}^{t} G(s) d s .
$$




\subsection{Statement of the result}

We will use the following hypotheses

$$
\begin{gathered}
U_{0} \in \mathcal{U}_{0}, \quad M_{0}, Q_{0} \in \mathbb{L}^{2}(D), \operatorname{div} Q_{0} \in L^{12 / 11}(D), \theta_{0} \in L^{4}(D) \\
g \in \mathbb{L}^{\infty}\left(D_{T}\right), \quad F \in H^{1}\left(0, T ; L^{2}(D)\right), \int_{D} F(t, x) d x=0 \text { for all } t \in[0, T] .
\end{gathered}
$$

Let us give now the definition of a global weak solution to problem $(\mathcal{P})$

Definition 1 We say that $(U, M, H, \theta, Q)$ is a global weak solution with finite energy of problem $(\mathcal{P})$ if the following conditions are fulfilled

$$
\begin{aligned}
& U \in L^{\infty}\left(0, T ; \mathcal{U}_{0}\right) \cap L^{2}(0, T ; \mathcal{U}) \\
& M \in L^{\infty}\left(0, T ; \mathbb{L}^{2}(D)\right) \cap L^{2}\left(0, T ; \mathbb{H}_{t}^{1}(D)\right) \\
& H \in L^{\infty}\left(0, T ; \mathbb{L}^{2}(D)\right) \cap L^{2}\left(0, T ; \mathbb{H}_{t}^{1}(D)\right) \\
& Q \in L^{\infty}\left(0, T ; \mathbb{L}^{2}(D)\right) \cap L^{2}\left(0, T ; \mathbb{H}_{n}^{1}(D)\right) \\
& \theta \in L^{\infty}\left(0, T ; L^{4}(D)\right)
\end{aligned}
$$

and

(i) the linear momentum equation holds weakly in the sense that for all $v \in \mathcal{U}$

$$
\begin{gathered}
\frac{d}{d t} \int_{D} U \cdot v d x+\int_{D}(U \cdot \nabla) U \cdot v d x+\eta \int_{D} \nabla U \cdot \nabla v d x= \\
-\int_{D} \rho(\theta) g \cdot v d x+\mu_{0} \int_{D}(M \cdot \nabla) H \cdot v d x+\frac{\mu_{0}}{2} \int_{D} M \times H \cdot \operatorname{curl} v d x \\
U(0)=U_{0}
\end{gathered}
$$

(ii) the magnetization equation satisfies for all $w \in \mathbb{H}_{t}^{1}(D)$ the weak formulation

$$
\begin{gathered}
\frac{d}{d t} \int_{D} M \cdot w d x+\int_{D}(U \cdot \nabla) M \cdot w d x+\sigma \int_{D} \operatorname{curl} U \cdot \operatorname{curl} w d x \\
+\sigma \int_{D} \operatorname{div} M \operatorname{div} w d x+\frac{1}{\delta} \int_{D}\left(M-\chi_{0} H\right) \cdot w d x= \\
\frac{1}{2} \int_{D} \operatorname{curl} U \times H \cdot w d x-\beta_{0} \int_{D} M \times H \cdot M \times w d x \\
M(0)=M_{0}
\end{gathered}
$$

(iii) the magnetic field is given by $H=\nabla \varphi$ where $\varphi \in L^{\infty}\left(0, T ; L_{\sharp}^{2}\right)$ and satisfies for all $\psi \in H_{\sharp}^{1}$

$$
\int_{D}(\nabla \varphi(t)+M(t)) \cdot \nabla \psi d x=-\int_{D} F(t) \psi d x
$$

(iv) the couple $(\theta, Q)$ satisfies the Maxwell-Cattaneo system in the following sense

$$
\int_{D_{T}} \theta\left(\partial_{t} a+U \cdot \nabla a\right) d x d t=\int_{D_{T}} \operatorname{div} Q a d x d t-\int_{D} \theta_{0} a(0) d x
$$


for all $a \in \mathcal{D}\left(\left[0, T[\times \bar{D})\right.\right.$ and for all $b \in \mathbb{H}_{n}^{1}(D)$ with $\operatorname{div} b \in L^{4}(D)$

$$
\begin{gathered}
\tau \frac{d}{d t} \int_{D_{T}} Q \cdot b d x+\tau \gamma \int_{D}(\operatorname{curl} Q \cdot \operatorname{curl} b+\operatorname{div} Q \operatorname{div} b) d x+ \\
\int_{D} Q \cdot b d x+\frac{\tau}{2} \int_{D} \operatorname{curl} U \times Q \cdot b d x=\int_{D} \mathcal{K}(\theta) \operatorname{div} b d x \\
Q(0)=Q_{0} .
\end{gathered}
$$

Moreover the energy estimates (28) and (31) hold for all $t \in(0, T)$.

\section{Remark 1}

1. As usual, we get the pressure $p \in W^{-1, \infty}\left(0, T ; L^{2}(D)\right)$ by using the De Rham theorem.

2. From the weak formulations, we deduce that $\left(\partial_{t} U, \partial_{t} M, \partial_{t} Q\right) \in L^{1}\left(0, T ; \mathcal{U}^{\prime} \times\right.$ $\left.\left(H_{t}^{1}(D)\right)^{\prime} \times\left(H_{n}^{1}(D)\right)^{\prime}\right)$ so that $(U, M, Q) \in \mathcal{C}\left([0, T] ; \mathcal{U}^{\prime} \times\left(H_{t}^{1}(D)\right)^{\prime} \times\left(H_{n}^{1}(D)\right)^{\prime}\right)$ and the corresponding initial conditions are meaningful and moreover $U, M, Q \in$ $\mathcal{C}\left([0, T] ; \mathbb{L}^{2}(D)\right.$ weak $)$.

3. The theory of transport equation leads to the result $\theta \in \mathcal{C}\left([0, T] ; \mathbb{L}^{4}(D)\right.$ weak $) \cap$ $\mathcal{C}\left([0, T] ; \mathbb{L}^{p}(D)\right), \quad$ for all $1 \leq p<4$ (see [5] for example) which gives a sense to the initial condition.

Theorem 1 Under hypotheses (36), there exists a global weak solution with finite energy of problem $(\mathcal{P})$. Moreover $\theta$ has the regularity

$$
\theta \in L^{36 / 11}\left(0, T ; L^{36 / 7}(D)\right)
$$

Remark 2 One can relax the condition $\operatorname{div} b \in L^{4}(D)$ on test functions $b$ in (42) to the condition $\operatorname{div} b \in L^{12 / 5}(D)$.

We will prove existence of solutions to problem $(\mathcal{P})$ in several steps, using a regularization method and some compactness results. The paper is organized as follows.

In section 3 , we introduce the regularized problem $\left(\mathcal{P}_{\nu}\right)$ obtained by adding an elliptic term $-\nu \nabla \cdot\left(|\nabla \theta|^{2} \nabla \theta\right)$ in the temperature equation, $\nu>0$ being a small parameter together to a regularization of the initial condition $\theta_{0}$. By using the Faedo-Galerkine method, we obtain a sequence of approximated solutions $\left(U_{n}, M_{n}, H_{n}, \theta_{n}, Q_{n}\right)$ which converge towards $\left(U_{\nu}, M_{\nu}, H_{\nu}, \theta_{\nu}, Q_{\nu}\right)$ a global weak solution with finite energy of system $\left(\mathcal{P}_{\nu}\right)$.

In section 4 , we prove Theorem 1 . We first introduce an auxiliary problem satisfied by $\zeta_{\nu}=\tau \gamma \operatorname{div} Q_{\nu}-\mathcal{K}\left(\theta_{\nu}\right)$ and establish a compacity result verified by $\zeta_{\nu}$ which allows to get the limit of the nonlinear term $\mathcal{K}\left(\theta_{\nu}\right)$. Then we get Theorem 1 by passing to the limit as $\nu \rightarrow 0$. 


\section{The regularized problem $\left(\mathcal{P}_{\nu}\right)$}

Let $\nu>0$ be a small parameter and $\left(\theta_{0}^{\nu}\right)$ such that

$$
\left(\theta_{0}^{\nu}\right) \subset W^{1,4}(D), \quad \theta_{0}^{\nu} \rightarrow \theta_{0} \text { strongly in } L^{4}(D) .
$$

We define the regularized problem $\left(\mathcal{P}_{\nu}\right)$ as the system $(8)-(12)$ coupled to the regularized Maxwell-Cattaneo system

$$
\begin{aligned}
& \partial_{t} \theta+(U \cdot \nabla) \theta-\nu \nabla \cdot\left(|\nabla \theta|^{2} \nabla \theta\right)=-\operatorname{div} Q \text { in } D_{T} \\
& \tau\left(\partial_{t} Q-\gamma \Delta Q\right)=-\frac{\tau}{2} \operatorname{curl} U \times Q-Q-\nabla \mathcal{K}(\theta) \text { in } D_{T} \\
& \nu|\nabla \theta|^{2} \nabla \theta \cdot \mathbf{n}=0, Q \times \mathbf{n}=0, \tau \gamma \operatorname{div} Q-\mathcal{K}(\theta)=0 \text { on } \Gamma_{T} \\
& \theta(0)=\theta_{0}^{\nu}, Q(0)=Q_{0} \text { in } D
\end{aligned}
$$

Note that we use the nonlinear elliptic operator $-\nu \nabla \cdot\left(|\nabla \theta|^{2} \nabla \theta\right)$ instead of $-\nu \Delta \theta$ which is commonly used to regularize a transport equation, owing to obtain approximate solutions $\theta_{\nu}$ belonging to $W^{1,4}(D)$ and therefore to $L^{\infty}(D)$.

Proceeding as previously the energy associated with (45) takes the form

$$
\mathcal{E}_{m c}(t)+\int_{0}^{t} \mathcal{F}_{m c}(s) d s+\nu \int_{0}^{t} \mathcal{R}(s) d s \leq \mathcal{E}_{m c, 0}^{\nu}
$$

for all $t \geq 0$ where

$$
\mathcal{R}(t)=\kappa\|\nabla \theta\|_{L^{4}(D)}^{4}+3 \alpha \int_{D} \theta^{2}|\nabla \theta|^{4} d x
$$

which is well defined thanks to the Sobolev embedding $W^{1,4}(D) \subset \mathcal{C}(\bar{D})$ and

$$
\mathcal{E}_{m c, 0}^{\nu}=\int_{D}\left(\frac{\kappa}{2}\left|\theta_{0}^{\nu}\right|^{2}+\int_{D} \frac{\alpha}{4}\left|\theta_{0}^{\nu}\right|^{4}\right) d x+\left\|Q_{0}\right\|^{2}
$$

It is easy to verify that the energy estimate associated with the problem $\left(\mathcal{P}_{\nu}\right)$ writes as

$$
\mathcal{E}(t)+\int_{0}^{t} \mathcal{F}(s) d s+\nu \int_{0}^{t} \mathcal{R}(s) d s \leq C+C \int_{0}^{t}\|\rho(\theta(s))\|^{2} d s+C \int_{0}^{t}\|G(s)\|^{2} d s
$$

where $C>0$ does not depend on $\nu$. We will prove the following existence result

Theorem 2 Under hypotheses (36), there exists a global weak solution $\left(U_{\nu}, M_{\nu}, H_{\nu}, \theta_{\nu}, Q_{\nu}\right)$ of problem $\left(\mathcal{P}_{\nu}\right)$ such that

$$
\begin{aligned}
& U_{\nu} \in L^{\infty}\left(0, T ; \mathcal{U}_{0}\right) \cap L^{2}(0, T ; \mathcal{U}) \\
& M_{\nu}, H_{\nu} \in L^{\infty}\left(0, T ; \mathbb{L}^{2}(D)\right) \cap L^{2}\left(0, T ; \mathbb{H}_{t}^{1}(D)\right) \\
& Q_{\nu} \in L^{\infty}\left(0, T ; \mathbb{L}^{2}(D)\right) \cap L^{2}\left(0, T ; \mathbb{H}_{n}^{1}(D)\right) \\
& \theta_{\nu} \in L^{\infty}\left(0, T ; L^{4}(D)\right) \cap L^{4}\left(0, T ; W^{1,4}(D)\right)
\end{aligned}
$$

and satisfying the energy estimates (46) and (48) and the problem in the following sense 
(i) $U_{\nu}(0)=U_{0}$ and for all $v \in \mathcal{U}$

$$
\begin{gathered}
\frac{d}{d t} \int_{D} U_{\nu} \cdot v d x+\int_{D}\left(U_{\nu} \cdot \nabla\right) U_{\nu} \cdot v d x+\eta \int_{D} \nabla U_{\nu} \cdot \nabla v d x= \\
-\int_{D} \rho\left(\theta_{\nu}\right) g \cdot v d x+\mu_{0} \int_{D}\left(M_{\nu} \cdot \nabla\right) H_{\nu} \cdot v d x+\frac{\mu_{0}}{2} \int_{D} M_{\nu} \times H_{\nu} \cdot \operatorname{curl} v d x
\end{gathered}
$$

(ii) $M_{\nu}(0)=M_{0}$ and for all $w \in \mathbb{H}_{t}^{1}(D)$

$$
\begin{gathered}
\frac{d}{d t} \int_{D} M_{\nu} \cdot w d x+\int_{D}\left(U_{\nu} \cdot \nabla\right) M_{\nu} \cdot w d x+\sigma \int_{D} \operatorname{curl} M_{\nu} \cdot \operatorname{curl} w d x \\
+\sigma \int_{D} \operatorname{div} M_{\nu} \operatorname{div} w d x+\frac{1}{\delta} \int_{D}\left(M_{\nu}-\chi_{0} H_{\nu}\right) \cdot w d x= \\
\frac{1}{2} \int_{D} \operatorname{curl} U_{\nu} \times H_{\nu} \cdot w d x-\beta_{0} \int_{D} M_{\nu} \times H_{\nu} \cdot M_{\nu} \times w d x
\end{gathered}
$$

(iii) $\theta_{\nu}(0)=\theta_{0}^{\nu}$ and for all $a \in W^{1,4}(D)$

$$
\frac{d}{d t} \int_{D} \theta_{\nu} a d x-\int_{D} \theta_{\nu} U \cdot \nabla a d x+\nu \int_{D}\left|\nabla \theta_{\nu}\right|^{2} \nabla \theta_{\nu} \cdot \nabla a d x=-\int_{D} \operatorname{div} Q_{\nu} a d x
$$

(iv) $Q_{\nu}(0)=Q_{0}$ and for all $b \in \mathbb{H}_{n}^{1}(D)$

$$
\begin{aligned}
& \tau \frac{d}{d t} \int_{D} Q_{\nu} \cdot b d x+\tau \gamma \int_{D}\left(\operatorname{curl} Q_{\nu} \cdot \operatorname{curl} b+\operatorname{div} Q_{\nu} \operatorname{div} b\right) d x+ \\
& \frac{\tau}{2} \int_{D} \operatorname{curl} U_{\nu} \times Q_{\nu} \cdot b d x=-\int_{D} Q_{\nu} \cdot b d x+\int_{D} \mathcal{K}\left(\theta_{\nu}\right) \operatorname{div} b d x
\end{aligned}
$$

with $H_{\nu}=\nabla \varphi_{\nu}$ where $\varphi_{\nu}=\mathcal{H}\left(M_{\nu}, F\right)$ is defined in (20).

\subsection{Faedo-Galerkine approximation for $\left(\mathcal{P}_{\nu}\right)$}

Let $\nu>0$ be fixed, consider the weak formulation of problem $\left(\mathcal{P}_{\nu}\right)$ given in Theorem 2 . In order to solve this problem by the Faedo-Galerkine method, we introduce the Hilbert basis $\left(V_{j}\right)_{j \geq 1},\left(W_{j}\right)_{j \geq 1},\left(\Phi_{j}\right)_{j \geq 1}$ of the spaces $\mathcal{U}, \mathbb{H}_{t}^{1}(D), \mathbb{H}_{n}^{1}(D)$ respectively and a basis $\left(v_{j}\right)_{j \geq 1}$ of $W^{1,4}(D)$. For simplicity, we assume these basis to be orthonormal in $L^{2}(D)$. We seek for approximated solutions of the system $\left(\mathcal{P}_{\nu}\right)$ of the form

$$
\begin{aligned}
U_{n}(t)=\sum_{j=1}^{n} \alpha_{j}(t) V_{j}, & M_{n}(t)=\sum_{j=1}^{n} \beta_{j}(t) W_{j}, \\
\theta_{n}(t)=\sum_{j=1}^{n} a_{j}(t) v_{j}, & Q_{n}(t)=\sum_{j=1}^{n} b_{j}(t) \Phi_{j}
\end{aligned}
$$

satisfying for all $n \in \mathbb{N}^{*}$ and $1 \leq j \leq n$

$$
\begin{gathered}
\text { (i) } \frac{d}{d t} \int_{D} U_{n} \cdot V_{j} d x+\int_{D}\left(U_{n} \cdot \nabla\right) U_{n} \cdot V_{j} d x+\eta \int_{D} \nabla U_{n} \cdot \nabla V_{j} d x= \\
-\int_{D} \rho\left(\theta_{n}\right) g \cdot V_{j} d x+\mu_{0} \int_{D}\left(M_{n} \cdot \nabla\right) H_{n} \cdot V_{j} d x+\frac{\mu_{0}}{2} \int_{D} M_{n} \times H_{n} \cdot \operatorname{curl} V_{j} d x \\
U_{n}(0)=U_{0 n}
\end{gathered}
$$


(ii) $\frac{d}{d t} \int_{D} M_{n} \cdot W_{j} d x+\int_{D}\left(U_{n} \cdot \nabla\right) M_{n} \cdot W_{j} d x+\sigma \int_{D} \operatorname{curl} M_{n} \cdot \operatorname{curl} W_{j} d x$

$$
\begin{gathered}
+\sigma \int_{D} \operatorname{div} M_{n} \operatorname{div} W_{j} d x+\frac{1}{\delta} \int_{D}\left(M_{n}-\chi_{0} H_{n}\right) \cdot W_{j} d x= \\
\frac{1}{2} \int_{D} \operatorname{curl} U_{n} \times H_{n} \cdot W_{j} d x-\beta_{0} \int_{D} M_{n} \times H_{n} \cdot M_{n} \times W_{j} d x \\
M_{n}(0)=M_{0 n}
\end{gathered}
$$

$$
\begin{gathered}
\frac{d}{d t} \int_{D} \theta_{n} v_{j} d x-\int_{D} \theta_{n} U_{n} \cdot \nabla v_{j} d x+\nu \int_{D}\left|\nabla \theta_{n}\right|^{2} \nabla \theta_{n} \cdot \nabla v_{j} d x= \\
-\int_{D} \operatorname{div} Q_{n} v_{j} d x \\
\theta_{n}(0)=\theta_{0 n}^{\nu}
\end{gathered}
$$

$$
\begin{gathered}
\tau \frac{d}{d t} \int_{D} Q_{n} \cdot \Phi_{j} d x+\tau \gamma \int_{D}\left(\operatorname{curl} Q_{n} \cdot \operatorname{curl} \Phi_{j}+\operatorname{div} Q_{n} \operatorname{div} \Phi_{j}\right) d x= \\
-\frac{\tau}{2} \int_{D} \operatorname{curl} U_{n} \times Q_{n} \cdot \Phi_{j} d x-\int_{D} Q_{n} \cdot \Phi_{j} d x+\int_{D} \mathcal{K}\left(\theta_{n}\right) \operatorname{div} \Phi_{j} d x \\
Q_{n}(0)=Q_{0 n}
\end{gathered}
$$

where

$$
\begin{gathered}
\left.H_{n}=\nabla \varphi_{n}, \quad \varphi_{n}=\mathcal{H}\left(M_{n}, F\right)\right) \\
U_{0 n}=\sum_{j=1}^{n} \alpha_{0 n}^{j} V_{j}, \quad M_{0 n}=\sum_{j=1}^{n} \beta_{0 n}^{j} W_{j}, \\
\theta_{0 n}^{\nu}=\sum_{j=1}^{n} a_{0 n}^{\nu, j} v_{j}, \quad Q_{0 n}=\sum_{j=1}^{n} b_{0 n}^{j} \Phi_{j} .
\end{gathered}
$$

We assume that

$$
\begin{gathered}
\left(U_{0 n}, M_{0 n}, Q_{0 n}\right) \rightarrow\left(U_{0}, M_{0}, Q_{0}\right) \text { strongly in }\left(\mathbb{L}^{2}(D)\right)^{3} \\
\theta_{0 n}^{\nu} \rightarrow \theta_{0}^{\nu} \text { strongly in } W^{1,4}(D) .
\end{gathered}
$$

This problem will be labeled $\left(\mathcal{P}_{\nu}^{n}\right)$.

\subsection{Solving the system $\left(\mathcal{P}_{\nu}^{n}\right)$}

Let the vector valued functions $\alpha^{n}=\left(\alpha_{1}, \cdots, \alpha_{n}\right), \beta^{n}=\left(\beta_{1}, \cdots, \beta_{n}\right), a^{n}=\left(a_{1}, \cdots, a_{n}\right)$ and $b^{n}=\left(b_{1}, \cdots, b_{n}\right)$, we consider the function

$$
t \in[0, T] \rightarrow \gamma_{n}(t)=\left(\alpha^{n}(t), \beta^{n}(t), a^{n}(t), b^{n}(t)\right) \in\left(\mathbb{R}^{n}\right)^{4}
$$

then $\gamma_{n}$ satisfies the ordinary differential system

$$
\gamma_{n}^{\prime}+A_{n} \gamma_{n}=Z_{n}\left(t, \gamma_{n}\right), \quad \gamma_{n}(0)=\gamma_{0 n}
$$

where $\gamma_{0 n}=\left(\alpha_{0 n}, \beta_{0 n}, a_{0 n}^{\nu}, b_{0 n}\right) \in\left(\mathbb{R}^{n}\right)^{4}, A_{n}$ is a $n^{4} \times n^{4}$ constant matrix involving the terms

$$
\begin{gathered}
\eta \int_{D} \nabla V_{i} \cdot \nabla V_{j} d x, \quad \sigma \int_{D}\left(\operatorname{curl} W_{i} \cdot \operatorname{curl} W_{j}+\operatorname{div} W_{i} \operatorname{div} W_{j}\right) d x \\
\tau \gamma \int_{D}\left(\operatorname{curl} \Phi_{i} \cdot \operatorname{curl} \Phi_{j}+\operatorname{div} \Phi_{i} \operatorname{div} \Phi_{j}\right) d x+\int_{D} \Phi_{i} \cdot \Phi_{j} d x
\end{gathered}
$$


and the vector field $Z_{n}=\left(Z_{n}^{1}, Z_{n}^{2}, Z_{n}^{3}, Z_{n}^{4}\right) \in\left(\mathbb{R}^{n}\right)^{4}$ is defined as follows

$$
\begin{gathered}
Z_{n j}^{1}\left(t, \gamma_{n}\right)=-\int_{D}\left(U_{n} \cdot \nabla\right) U_{n} \cdot V_{j} d x-\int_{D} \rho\left(\theta_{n}\right) g \cdot V_{j} d x \\
+\mu_{0} \int_{D}\left(U_{n} \cdot \nabla\right) H_{n} \cdot V_{j} d x+\frac{\mu_{0}}{2} \int_{D} M_{n} \times H_{n} \cdot \operatorname{curl} V_{j} d x \\
Z_{n j}^{2}\left(t, \gamma_{n}\right)=-\int_{D}\left(U_{n} \cdot \nabla\right) M_{n} \cdot W_{j} d x-\frac{1}{\delta} \int_{D}\left(M_{n}-\chi_{0} H_{n}\right) \cdot W_{j} d x \\
+\frac{1}{2} \int_{D} \operatorname{curl} U_{n} \times H_{n} \cdot W_{j} d x-\beta_{0} \int_{D} M_{n} \times H_{n} \cdot M_{n} \times W_{j} d x \\
Z_{n j}^{3}\left(t, \gamma_{n}\right)=\int_{D} \theta_{n} U_{n} \cdot \nabla v_{j} d x-\nu \int_{D}\left|\nabla \theta_{n}\right|^{2} \nabla \theta_{n} \cdot \nabla v_{i} d x-\int_{D} \operatorname{div} Q_{n} v_{j} d x \\
Z_{n j}^{4}\left(t, \gamma_{n}\right)=-\frac{\tau}{2} \int_{D} \operatorname{curl} U_{n} \times Q_{n} \cdot \Phi_{j} d x+\int_{D} \mathcal{K}\left(\theta_{n}\right) \operatorname{div} \Phi_{j} d x
\end{gathered}
$$

for $1 \leq j \leq n$.

Notice that $Z_{n}$ has the same regularity in the time variable $t$ as the function $F$ appearing in the magnetostatic equation and it is continuous and locally lipschitz continuous with respect to the variable $\gamma_{n}$. Hence there exists a unique maximal solution $\gamma_{n}$ of (60) defined on a time interval $\left[0 ; T_{n}\right]$ satisfying $\gamma_{n} \in H^{1}\left(0, T_{n} ;\left(\mathbb{R}^{n}\right)^{4}\right)$. We shall prove that $T_{n}=T$ with the following estimate.

Let $\left(U_{n}, M_{n}, \theta_{n}, Q_{n}\right)$ be the solution of $\left(\mathcal{P}_{\nu}^{n}\right)$ defined on $\left(0, T_{n}\right)$. We want to verify that

$$
\sup _{t \in\left[0 ; T_{n}\right]}\left(\left\|U_{n}\right\|^{2}+\left\|M_{n}\right\|^{2}+\left\|\theta_{n}\right\|_{L^{4}(D)}^{4}+\left\|Q_{n}\right\|^{2}\right)(t)<\infty .
$$

We multiply equation (58) by $b_{j}$ and add these equations for $1 \leq j \leq n$, we obtain

$$
\frac{\tau}{2} \frac{d}{d t}\left\|Q_{n}\right\|^{2}+\tau \gamma\left(\left\|\operatorname{curl} Q_{n}\right\|^{2}+\left\|\operatorname{div} Q_{n}\right\|^{2}\right)+\left\|Q_{n}\right\|^{2}=\int_{D} \mathcal{K}\left(\theta_{n}\right) \operatorname{div} Q_{n} d x
$$

We use the equation (57) that we multiply by $\Theta_{j}(t)=\int_{D} \mathcal{K}\left(\theta_{n}\right) \cdot v j d x$ and add the equalities for $1 \leq j \leq n$ to obtain

$$
\begin{gathered}
\frac{d}{d t}\left(\frac{\kappa}{2}\left\|\theta_{n}\right\|^{2}+\frac{\alpha}{4}\left\|\theta_{n}\right\|_{L^{4}(D)}^{4}\right)+\nu\left(\kappa\left\|\nabla \theta_{n}\right\|_{L^{4}(D)}^{4}+3 \alpha \int_{D} \theta_{n}^{2}\left|\nabla \theta_{n}\right|^{4} d x\right)= \\
-\int_{D} \operatorname{div} Q_{n} \mathcal{K}\left(\theta_{n}\right) d x .
\end{gathered}
$$

Adding (62) and (63) lead to

$$
\begin{gathered}
\frac{1}{2} \frac{d}{d t}\left(\kappa\left\|\theta_{n}\right\|^{2}+\frac{\alpha}{2}\left\|\theta_{n}\right\|_{L^{4}(D)}^{4}+\tau\left\|Q_{n}\right\|^{2}\right)+\nu \kappa\left\|\nabla \theta_{n}\right\|_{L^{4}(D)}^{4} \\
+3 \nu \alpha\left\|\theta_{n}\left|\nabla \theta_{n}\right|^{2}\right\|^{2}+\tau \gamma\left(\left\|\operatorname{curl} Q_{n}\right\|^{2}+\left\|\operatorname{div} Q_{n}\right\|^{2}\right)+\left\|Q_{n}\right\|^{2}=0
\end{gathered}
$$

Therefore, integrating between 0 and $t$ and using (59), we easily deduce that

$$
\begin{gathered}
\left(\kappa\left\|\theta_{n}\right\|^{2}+\frac{\alpha}{2}\left\|\theta_{n}\right\|_{L^{4}(D)}^{4}+\tau\left\|Q_{n}\right\|^{2}\right)(t)+2 \nu \int_{0}^{t} \kappa\left\|\nabla \theta_{n}\right\|_{L^{4}(D)}^{4} d s \\
+2 \int_{0}^{t}\left(3 \nu \alpha\left\|\theta_{n}\left|\nabla \theta_{n}\right|^{2}\right\|^{2}+\tau \gamma\left(\left\|\operatorname{curl} Q_{n}\right\|^{2}+\left\|\operatorname{div} Q_{n}\right\|^{2}\right)+\left\|Q_{n}\right\|^{2}\right) d s= \\
\kappa\left\|\theta_{0 n}^{\nu}\right\|^{2}+\frac{\alpha}{2}\left\|\theta_{0 n}^{\nu}\right\|_{L^{4}(D)}^{4}+\tau\left\|Q_{0 n}\right\|^{2} \leq C
\end{gathered}
$$


with $C$ independent of $n$. Similarly, we obtain from equations (55) and (56)

$$
\begin{gathered}
\frac{1}{2} \frac{d}{d t}\left\|U_{n}\right\|^{2}+\eta\left\|\nabla U_{n}\right\|^{2}=-\int_{D} \rho\left(\theta_{n}\right) g \cdot U_{n} d x \\
-\mu_{0} \int_{D}\left(U_{n} \cdot \nabla\right) M_{n} \cdot H_{n} d x+\frac{\mu_{0}}{2} \int_{D}\left(M_{n} \times H_{n}\right) \cdot \operatorname{curl} U_{n} d x \\
\frac{1}{2} \frac{d}{d t}\left\|M_{n}\right\|^{2}+\sigma\left\|\operatorname{curl} M_{n}\right\|^{2}+\sigma\left\|\operatorname{div} M_{n}\right\|^{2}+\frac{1}{\delta}\left\|M_{n}\right\|^{2}= \\
+\frac{\chi_{0}}{\delta} \int_{D} H_{n} \cdot M_{n} d x+\frac{1}{2} \int_{D} \operatorname{curl} U_{n} \times H_{n} \cdot M_{n} d x
\end{gathered}
$$

so (66) and (67) lead to

$$
\begin{gathered}
\frac{1}{2} \frac{d}{d t}\left(\left\|U_{n}\right\|^{2}+\mu_{0}\left\|M_{n}\right\|^{2}\right)+\eta\left\|\nabla U_{n}\right\|^{2}+\mu_{0} \sigma\left(\left\|\operatorname{curl} M_{n}\right\|^{2}+\left\|\operatorname{div} M_{n}\right\|^{2}\right)+\frac{\mu_{0}}{\delta}\left\|M_{n}\right\|^{2} \\
=-\int_{D} \rho\left(\theta_{n}\right) g \cdot U_{n} d x-\mu_{0} \int_{D}\left(U_{n} \cdot \nabla\right) M_{n} \cdot H_{n} d x+\frac{\mu_{0} \chi_{0}}{\delta} \int_{D} H_{n} \cdot M_{n} d x
\end{gathered}
$$

Using equation (24) for unknown $\varphi_{n}$ and data $M_{n}$, and testing with $\psi=\varphi_{n}$, we get

$$
\int_{D} H_{n} \cdot \partial_{t} M_{n} d x=-\frac{1}{2} \frac{d}{d t}\left\|H_{n}\right\|^{2}-\int_{D} \partial_{t} F \varphi_{n} d x
$$

Now we multiply equation $(56)$ by $h_{j}(t)=\int_{D} H_{n} \cdot W_{j} d x$ and add the equalities for $1 \leq j \leq n$ to obtain

$$
\begin{gathered}
\int_{D} \partial_{t} M_{n} \cdot H_{n} d x+\int_{D}\left(U_{n} \cdot \nabla\right) M_{n} \cdot H_{n} d x+\sigma \int_{D} \operatorname{div} M_{n} \operatorname{div} H_{n} d x \\
+\frac{1}{\delta} \int_{D}\left(M_{n}-\chi_{0} H_{n}\right) \cdot H_{n} d x=-\beta_{0}\left\|M_{n} \times H_{n}\right\|^{2}
\end{gathered}
$$

so

$$
\begin{gathered}
\int_{D}\left(U_{n} \cdot \nabla\right) M_{n} \cdot H_{n} d x=\frac{1}{2} \frac{d}{d t}\left\|H_{n}\right\|^{2}+\int_{D} \partial_{t} F \varphi_{n} d x-\frac{1}{\delta} \int_{D} M_{n} \cdot H_{n} d x \\
-\sigma \int_{D} \operatorname{div} M_{n}\left(F-\operatorname{div} M_{n}\right) d x+\frac{\chi_{0}}{\delta}\left\|H_{n}\right\|^{2}-\beta_{0}\left\|M_{n} \times H_{n}\right\|^{2} .
\end{gathered}
$$

$>$ From (21), we see that

$$
\int_{D} H_{n} \cdot M_{n} d x=-\left\|H_{n}\right\|^{2}-\int_{D} F \varphi_{n} d x
$$

therefore

$$
\begin{gathered}
\int_{D}\left(U_{n} \cdot \nabla\right) M_{n} \cdot H_{n} d x=\frac{1}{2} \frac{d}{d t}\left\|H_{n}\right\|^{2}+\int_{D}\left(\partial_{t} F+\frac{1}{\delta} F\right) \varphi_{n} d x+\sigma\left\|\operatorname{div} M_{n}\right\|^{2} \\
-\sigma \int_{D} \operatorname{div} M_{n} F d x+\frac{1+\chi_{0}}{\delta}\left\|H_{n}\right\|^{2}-\beta_{0}\left\|M_{n} \times H_{n}\right\|^{2} .
\end{gathered}
$$


so integrating between 0 and $t$, we get

$$
\begin{gathered}
\frac{1}{2}\left(\left\|U_{n}\right\|^{2}+\mu_{0}\left(\left\|M_{n}\right\|^{2}+\left\|H_{n}\right\|^{2}\right)(t)+\eta \int_{0}^{t}\left\|\nabla U_{n}\right\|^{2} d s\right. \\
+\int_{0}^{t}\left(\mu_{0} \sigma\left(\left\|\operatorname{curl} M_{n}\right\|^{2}+2\left\|\operatorname{div} M_{n}\right\|^{2}\right)+\frac{\mu_{0}}{\delta}\left\|M_{n}\right\|^{2}\right) d s \\
+\int_{0}^{t} \frac{\mu_{0}\left(1+2 \chi_{0}\right)}{\delta}\left\|H_{n}\right\|^{2} d s+\beta_{0} \mu_{0} \int_{0}^{t}\left\|M_{n} \times H_{n}\right\|^{2} d s= \\
\frac{1}{2}\left(\left\|U_{0 n}\right\|^{2}+\mu_{0}\left(\left\|M_{0 n}\right\|^{2}+\left\|H_{0 n}\right\|^{2}\right)+\right. \\
\int_{0}^{t} \int_{D} \rho\left(\theta_{n}\right) g \cdot U_{n} d x d s-\frac{\mu_{0}\left(1+\chi_{0}\right)}{\delta} \int_{0}^{t} \int_{D} F \varphi_{n} d x d s \\
-\mu_{0} \int_{0}^{t} \int_{D} \partial_{t} F \varphi_{n} d x d s+\mu_{0} \sigma \int_{0}^{t} \int_{D} F \operatorname{div} M_{n} d x d s
\end{gathered}
$$

where

$$
H_{0 n}=\nabla \varphi_{0 n}, \quad \varphi_{0 n}=\mathcal{H}\left(M_{0 n}, F(0)\right) .
$$

Using the inequalities

$$
\begin{gathered}
\left|\int_{0}^{t} \int_{D} \rho\left(\theta_{n}\right) g \cdot U_{n} d x d s\right| \leq C_{T}+C \int_{0}^{t}\left\|\theta_{n}\right\|^{2} d s+\frac{1}{2} \int_{0}^{t}\left\|U_{n}\right\|^{2} d s \\
\frac{\mu_{0}\left(1+\chi_{0}\right)}{\delta}\left|\int_{0}^{t} \int_{D} F \varphi_{n} d x d s\right|+\mu_{0}\left|\int_{0}^{t} \int_{D} \partial_{t} F \varphi_{n} d x d s\right| \leq \\
C\left(\|F\|_{L^{2}\left(D_{T}\right)}^{2}+\left\|\partial_{t} F\right\|_{L^{2}\left(D_{T}\right)}^{2}\right)+\frac{\mu_{0}\left(1+2 \chi_{0}\right)}{2 \delta} \int_{0}^{t}\left\|H_{n}\right\|^{2} d s \\
\mu_{0} \sigma\left|\int_{0}^{t} \int_{D} F \operatorname{div} M_{n} d x d s\right| \leq C\|F\|_{L^{2}\left(D_{T}\right)}^{2}+\mu_{0} \sigma \int_{0}^{t}\left\|\operatorname{div} M_{n}\right\|^{2} d s .
\end{gathered}
$$

We get

$$
\begin{gathered}
\frac{1}{2}\left(\left\|U_{n}\right\|^{2}+\mu_{0}\left(\left\|M_{n}\right\|^{2}+\left\|H_{n}\right\|^{2}\right)(t)+\right. \\
\int_{0}^{t}\left[\eta\left\|\nabla U_{n}\right\|^{2}+\mu_{0} \sigma\left(\left\|\operatorname{curl} M_{n}\right\|^{2}+2\left\|\operatorname{div} M_{n}\right\|^{2}\right)+\frac{\mu_{0}}{\delta}\left\|M_{n}\right\|^{2}\right] d s \\
+\int_{0}^{t}\left[\frac{\mu_{0}\left(1+2 \chi_{0}\right)}{2 \delta}\left\|H_{n}\right\|^{2}+\beta_{0} \mu_{0}\left\|M_{n} \times H_{n}\right\|^{2}\right] d s \leq \\
A_{n}+C_{T}+C \int_{0}^{t}\left\|\theta_{n}\right\|^{2} d s+\frac{1}{2} \int_{0}^{t}\left\|U_{n}\right\|^{2} d s
\end{gathered}
$$

where

$$
A_{n}=\frac{1}{2}\left(\left\|U_{0 n}\right\|^{2}+\mu_{0}\left(\left\|M_{0 n}\right\|^{2}+\left\|H_{0 n}\right\|^{2}\right) \leq C\right.
$$


with $C$ independent of $n$ in view of (59), (72) and (19). Thus thanks to (65) and Gronwall inequality, we deduce that

$$
\left\|U_{n}(t)\right\|^{2}+\left\|M_{n}(t)\right\|^{2}+\left\|H_{n}(t)\right\|^{2} \leq C+\exp (C t)
$$

then

$$
\begin{gathered}
\frac{1}{2}\left(\left\|U_{n}\right\|^{2}+\mu_{0}\left(\left\|M_{n}\right\|^{2}+\left\|H_{n}\right\|^{2}\right)(t)+\right. \\
\int_{0}^{t}\left[\eta\left\|\nabla U_{n}\right\|^{2}+\mu_{0} \sigma\left(\left\|\operatorname{curl} M_{n}\right\|^{2}+2\left\|\operatorname{div} M_{n}\right\|^{2}\right)+\frac{\mu_{0}}{\delta}\left\|M_{n}\right\|^{2}\right] d s \\
+\int_{0}^{t}\left[\frac{\mu_{0}\left(1+2 \chi_{0}\right)}{2 \delta}\left\|H_{n}\right\|^{2}+\beta_{0} \mu_{0}\left\|M_{n} \times H_{n}\right\|^{2}\right] d s \leq C+\exp (C t) .
\end{gathered}
$$

This ends the proof of (61) so we conclude that $T_{n}=T$ for all $n \geq 1$.

\subsection{Convergence of the Faedo-Galerkine scheme}

Let $\nu$ be fixed, the estimates (65) and (75) show that

\section{Lemma 1}

- $\left(U_{n}\right)_{n}$ is uniformly bounded in $L^{\infty}\left(0, T ; \mathcal{U}_{0}\right) \cap L^{2}(0, T ; \mathcal{U})$

- $\left(M_{n}\right)_{n}$ and $H_{n}$ are uniformly bounded in $L^{\infty}\left(0, T ; \mathbb{L}^{2}(D)\right) \cap L^{2}\left(0, T ; \mathbb{H}_{t}^{1}(D)\right)$

- $\left(M_{n} \times H_{n}\right)_{n}$ is uniformly bounded in $L^{2}\left(0, T ; \mathbb{L}^{2}(D)\right)$

- $\left(Q_{n}\right)_{n}$ is uniformly bounded in $L^{\infty}\left(0, T ; \mathbb{L}^{2}(D)\right) \cap L^{2}\left(0, T ; \mathbb{H}_{n}^{1}(D)\right)$

- $\left(\theta_{n}\right)_{n}$ is uniformly bounded in $L^{\infty}\left(0, T ; L^{4}(D)\right) \cap L^{4}\left(0, T ; W^{1,4}(D)\right)$.

Notice that we get the uniform bound of $\left(H_{n}\right)_{n}$ in $L^{2}\left(0, T ; \mathbb{H}_{t}^{1}(D)\right)$ using the bound of $\left(M_{n}\right)_{n}$ and (23). Hence we get the convergence

Lemma 2 Let $\nu>0$ be fixed. There exists subsequences still denoted $\left(U_{n}\right),\left(M_{n}\right)$, $\left(H_{n}\right),\left(Q_{n}\right)$ and $\left(\theta_{n}\right)$ such that when $n \rightarrow \infty$

$U_{n} \rightarrow U_{\nu}$ weakly $-\star$ in $L^{\infty}\left(0, T ; \mathbb{L}^{2}(D)\right)$ and weakly in $L^{2}(0, T ; \mathcal{U})$

$M_{n} \rightarrow M_{\nu}, H_{n} \rightarrow H_{\nu}$ weakly $-\star$ in $L^{\infty}\left(0, T ; \mathbb{L}^{2}(D)\right)$ and weakly in $L^{2}\left(0, T ; \mathbb{H}_{t}^{1}(D)\right)$

$Q_{n} \rightarrow Q_{\nu}$ weakly $-\star$ in $L^{\infty}\left(0, T ; \mathbb{L}^{2}(D)\right)$ and weakly in $L^{2}\left(0, T ; \mathbb{H}_{n}^{1}(D)\right)$

$\theta_{n} \rightarrow \theta_{\nu}$ weakly $-\star$ in $L^{\infty}\left(0, T ; L^{4}(D)\right)$ and weakly in $L^{4}\left(0, T ; W^{1,4}(D)\right)$

Moreover, we have

$$
\left|\nabla \theta_{n}\right|^{2} \nabla \theta_{n} \rightarrow \Lambda_{\nu} \text { weakly in } L^{\frac{4}{3}}\left(D_{T}\right) .
$$

In order to pass to the limit in the nonlinear terms, we need strong convergence for the sequences in some spaces. To apply compactness results, we need to estimate the time derivatives of the solutions.

Let us begin with $\left(\partial_{t} \theta_{n}\right)_{n}$. We multiply equation (57) by $a_{j}^{\prime}(t)$ and add the resulting equalities for $1 \leq j \leq n$ to get

$$
\left\|\partial_{t} \theta_{n}\right\|^{2}+\int_{D} \partial_{t} \theta_{n} \nabla \theta_{n} \cdot U_{n} d x+\frac{\nu}{4} \frac{d}{d t}\left\|\nabla \theta_{n}\right\|_{L^{4}(D)}^{4}=-\int_{D} \operatorname{div} Q_{n} \partial_{t} \theta_{n}
$$


Since we have $\left|\int_{D} \partial_{t} \theta_{n} \nabla \theta_{n} \cdot U_{n} d x\right| \leq \frac{1}{4}\left\|\partial_{t} \theta_{n}\right\|^{2}+\left\|\nabla \theta_{n}\right\|_{L^{4}(D)}^{2}\left\|U_{n}\right\|_{L^{4}(D)}^{2}$ we obtain

$$
\left\|\partial_{t} \theta_{n}\right\|^{2}+\frac{\nu}{2} \frac{d}{d t}\left\|\nabla \theta_{n}\right\|_{L^{4}(D)}^{4} \leq 2\left\|\operatorname{div} Q_{n}\right\|^{2}+2\left\|\nabla \theta_{n}\right\|_{L^{4}(D)}^{2}\left\|U_{n}\right\|_{L^{4}(D)}^{2}
$$

Therefore, integrating between 0 and $t$, using (59) and (65), we easily deduce that

$$
\begin{gathered}
\int_{0}^{t}\left\|\partial_{t} \theta_{n}\right\|^{2} d s+\frac{\nu}{2}\left\|\nabla \theta_{n}\right\|_{L^{4}(D)}^{4} \leq \frac{\nu}{2}\left\|\nabla \theta_{0 n}^{\nu}\right\|_{L^{4}(D)}^{4}+ \\
2 \int_{0}^{T}\left\|\operatorname{div} Q_{n}\right\|^{2} d s+2 \int_{0}^{t}\left\|\nabla \theta_{n}\right\|_{L^{4}(D)}^{2}\left\|U_{n}\right\|_{L^{4}(D)}^{2} d s \\
\leq C+2 \int_{0}^{t}\left\|\nabla \theta_{n}\right\|_{L^{4}(D)}^{2}\left\|U_{n}\right\|_{L^{4}(D)}^{2} d s
\end{gathered}
$$

with $C$ independent of $n$.

Setting $y(t)=\left\|\nabla \theta_{n}(t)\right\|_{L^{4}(D)}^{4}$ and $F(t)=\left\|U_{n}\right\|_{L^{4}(D)}^{2}$ then from (79), $y(t)$ satisfies the integral inequality

$$
y(t) \leq C_{\nu}+2 M_{\nu} \int_{0}^{t} \sqrt{y(s)} F(s) d s .
$$

Using the Gronwall-Bellman-Bihari inequality (see [3]) we deduce

$$
y(t) \leq\left(\sqrt{C_{\nu}}+M_{\nu} \int_{0}^{t} F(s) d s\right)^{2} .
$$

Hence we get for all $t \in[0, T]$ the estimate

$$
\left\|\nabla \theta_{n}(t)\right\|_{L^{4}(D)}^{2} \leq \sqrt{C_{\nu}}+M_{\nu} \int_{0}^{t}\left\|U_{n}(s)\right\|_{L^{4}(D)}^{2} d s
$$

which leads to

$$
\int_{0}^{T}\left\|\partial_{t} \theta_{n}\right\|^{2} d s \leq C+2 \sqrt{C_{\nu}} \int_{0}^{T}\left\|U_{n}(s)\right\|_{L^{4}(D)}^{2} d s+2 M_{\nu}\left(\int_{0}^{T}\left\|U_{n}(s)\right\|_{L^{4}(D)}^{2} d s\right)^{2}
$$

and we conclude that $\left(\partial_{t} \theta_{n}\right)_{n}$ is uniformly bounded in $L^{2}\left(D_{T}\right)$ with respect to $n$.

To estimate $\partial_{t} U_{n}, \partial_{t} M_{n}$ and $\partial_{t} Q_{n}$ we need some notations. For a function $f$ defined on $[0, T]$ with values in a space $V$, let $\widetilde{f}$ be the function equal to $f$ on $[0, T]$ and to 0 elsewhere and let $\widehat{f}$ be its Fourier transform defined by

$$
\widehat{f}(\tau)=\int_{\mathbb{R}} \exp (-2 i \pi t \tau) \widetilde{f}(t) d t=\int_{0}^{T} \exp (-2 i \pi t \tau) f(t) d t, \quad \tau \in \mathbb{R} .
$$

We will prove that for $0<\gamma<1 / 4$,

$$
\int_{\mathbb{R}}|\tau|^{2 \gamma}\left\|\widehat{U}_{n}(\tau)\right\|^{2} d \tau \leq C .
$$

Proceeding as in [22] (see also [16]) and since $\left(U_{n}\right)_{n}$ is uniformly bounded in $L^{2}(0, T ; \mathcal{U})$, it is enough to verify that

$$
|\tau|\left\|\widehat{U}_{n}(\tau)\right\|^{2} \leq C\left\|\widehat{U}_{n}(\tau)\right\|_{\mathcal{U}}+C\left\|\widehat{U}_{n}(\tau)\right\|, \quad \forall \tau \in \mathbb{R}
$$


We write the equation (55) of $U_{n}$ in the form

$$
\frac{d}{d t} \int_{D} U_{n} \cdot V_{j} d x=\left\langle\mathcal{L}_{n}, V_{j}\right\rangle, \quad U_{n}(0)=U_{0 n}
$$

for all $1 \leq j \leq n$ where the linear form $\mathcal{L}_{n}$ is defined on $\mathcal{U}$ by

$$
\begin{aligned}
\left\langle\mathcal{L}_{n}, \varphi\right\rangle & =\int_{D}\left(U_{n} \cdot \nabla\right) U_{n} \cdot \varphi d x+\eta \int_{D} \nabla U_{n} \cdot \nabla \varphi d x+\int_{D} \rho\left(\theta_{n}\right) g \cdot \varphi d x \\
& -\mu_{0} \int_{D}\left(M_{n} \cdot \nabla\right) H_{n} \cdot \varphi d x-\frac{\mu_{0}}{2} \int_{D} M_{n} \times H_{n} \cdot \operatorname{curl} \varphi d x
\end{aligned}
$$

We have $\mathcal{L}_{n} \in \mathcal{U}^{\prime}$ p.p. $t \in(0, T)$ and

$$
\begin{gathered}
\left\|\mathcal{L}_{n}\right\|_{\mathcal{U}^{\prime}} \leq C\left(\left\|U_{n}\right\|_{H^{1}(D)}^{2}+\left\|U_{n}\right\|_{H^{1}(D)}+\left\|\rho\left(\theta_{n}\right)\right\|+\right. \\
\left.\left\|M_{n}\right\|_{H^{1}(D)}\left\|H_{n}\right\|_{H^{1}(D)}+\left\|M_{n} \times H_{n}\right\|\right)
\end{gathered}
$$

and we conclude thanks to Lemma 1 that $\left(\mathcal{L}_{n}\right)$ is uniformly bounded in $L^{1}\left(0, T ; \mathcal{U}^{\prime}\right)$. Now we rewrite (99) as follows

$$
\frac{d}{d t} \int_{D} \widetilde{U}_{n} \cdot V_{j} d x=\left\langle\widetilde{\mathcal{L}_{n}}, V_{j}\right\rangle+\left(\int_{D} U_{0 n} \cdot V_{j} d x\right) \delta_{0}-\left(\int_{D} U_{n}(T) \cdot V_{j} d x\right) \delta_{T}
$$

for $1 \leq j \leq n$ where $\delta_{a}$ denotes the Dirac distribution at $a \in \mathbb{R}$. Therefore, we obtain

$$
2 i \pi \tau \int_{D} \widehat{U}_{n} \cdot V_{j} d x=\left\langle\widehat{\mathcal{L}_{n}}, v_{j}\right\rangle+\int_{D} U_{0 n} \cdot V_{j} d x-\exp (-2 i \pi T \tau) \int_{D} U_{n}(T) \cdot V_{j} d x
$$

Next we multiply equality (86) by $\widehat{\widehat{\alpha_{j}}}(\tau)$ the conjugate of $\widehat{\alpha_{j}}(\tau)$ and add the equalities for $1 \leq j \leq n$ to get

$$
2 i \pi \tau\left\|\widehat{U}_{n}\right\|^{2}=\left\langle\widehat{\mathcal{L}_{n}}, \overline{\widehat{U}_{n}}\right\rangle+\int_{D} U_{0 n} \cdot \overline{\widehat{U}_{n}} d x-\exp (-2 i \pi T \tau) \int_{D} U_{n}(T) \cdot \overline{\widehat{U}_{n}} d x
$$

therefore since for all $\tau \in \mathbb{R}$, we have

$$
\left\|\widehat{\mathcal{L}_{n}}(\tau)\right\|_{\mathcal{U}^{\prime}} \leq \int_{0}^{T}\left\|\mathcal{L}_{n}(t)\right\|_{\mathcal{U}^{\prime}} d t \leq C
$$

then using Plancherel identity we get (82).

Similar proofs work for $\partial_{t} M_{n}$ and $\partial_{t} Q_{n}$. The above results are summarized in

Lemma 3 There exists $C_{\nu}>0$ such that for all $n$

$$
\int_{\mathbb{R}}|\tau|^{2 \gamma}\left(\left\|\widehat{U}_{n}(\tau)\right\|^{2}+\left\|\widehat{M}_{n}(\tau)\right\|^{2}+\left\|\widehat{Q}_{n}(\tau)\right\|^{2}\right) d \tau \leq C_{\nu}
$$

Moreover we have

$$
\left\|\partial_{t} \theta_{n}\right\|_{L^{2}\left(D_{T}\right)} \leq C_{\nu}
$$

Combining the bounds of Lemma 1 and Lemma 3 and applying Lions compactness lemma for $\left(U_{n}, M_{n}, Q_{n}\right)$ and and Aubin compactness lemma for $\theta_{n}$ we get the strong convergence results we get the strong convergence results 
Lemma 4 For $\nu>0$ fixed, we have

$$
\left(U_{n}, M_{n}, H_{n}, \theta_{n}, Q_{n}\right) \rightarrow\left(U_{\nu}, M_{\nu}, H_{\nu}, \theta_{\nu}, Q_{\nu}\right) \text { strongly in }\left(\mathbb{L}^{2}\left(D_{T}\right)\right)^{5}
$$

Moreover we have $H_{\nu}=\mathcal{H}\left(M_{\nu}, F\right)$.

The strong convergence of $\left(H_{n}\right)_{n}$ is a consequence of the continuity of operator $\mathcal{H}$ (see subsection 1.3).

Thanks to Lemma 2 and Lemma 4, we can pass to the limit in problem $\left(\mathcal{P}_{\nu}^{n}\right)$ when $n \rightarrow \infty$. We get that $\left(U_{\nu}, M_{\nu}, H_{\nu}, \theta_{\nu}, Q_{\nu}\right)$ satisfies the equations of system $\left(\mathcal{P}_{\nu}\right)$ except for the temperature equation satisfied by $\theta_{\nu}$ for which we obtain

$$
\frac{d}{d t} \int_{D} \theta_{\nu} a d x-\int_{D} \theta_{\nu} U_{\nu} \cdot \nabla a d x+\nu \int_{D} \Lambda_{\nu} \cdot \nabla a d x=-\int_{D} \operatorname{div} Q_{\nu} a
$$

for all $a \in W^{1,4}(D)$ and $\Lambda_{\nu}$ being defined in (76).

Passing to the limit in the temperature equation. Hereafter, we detail the procedure of passing to the limit in the equation of $\theta_{n}$. First we introduce some notations. Let $W=W^{1,4}(D)$ and $A$ the nonlinear operator defined on $W$ by

$$
\langle A(\varphi), \psi\rangle=\int_{D}|\nabla \varphi|^{2} \nabla \varphi \cdot \nabla \psi d x, \quad \forall \varphi, \psi \in W
$$

then $A(\varphi) \in W^{\prime}$ for all $\varphi \in W$ and

$$
\|A(\varphi)\|_{W^{\prime}} \leq\|\nabla \varphi\|_{L^{4}(D)}^{3} .
$$

Next we define on $\mathbb{L}^{2}(D) \times L^{4}(D)$ the bilinear operator $B$ by

$$
\langle B(\xi, \varphi), \psi\rangle=\int_{D} \varphi \xi \cdot \nabla \psi d x, \quad \forall \xi \in \mathbb{L}^{2}(D), \varphi \in L^{4}(D), \psi \in W .
$$

It holds that $B(\xi, \varphi) \in W^{\prime}$ for all $(\xi, \varphi) \in \mathbb{L}^{2}(D) \times L^{4}(D)$ and

$$
\|B(\xi, \varphi)\|_{W^{\prime}} \leq\|\varphi\|_{L^{4}(D)}\|\xi\|
$$

and we have $L^{2}(D) \subset W^{\prime}$ with

$$
\|f\|_{W^{\prime}} \leq(\operatorname{mes}(D))^{1 / 4}\|f\|, \quad \forall f \in L^{2}(D) .
$$

We multiply equation (57) by a function $f \in \mathcal{C}^{1}([0, T])$ such that $f(T)=0$ and integrate by parts. Then integrating by parts, we get for all $1 \leq j \leq n$

$$
\begin{aligned}
& -\int_{D_{T}} f^{\prime}(t) \theta_{n} v_{j} d x d t-f(0) \int_{D} \theta_{0 n}^{\nu} v_{j} d x-\int_{D_{T}} f(t) \theta_{n} U_{n} \cdot \nabla v_{j} d x d t \\
& \quad+\nu \int_{D_{T}} f(t)\left|\nabla \theta_{n}\right|^{2} \nabla \theta_{n} \cdot \nabla v_{j} d x d t=-\int_{D_{T}} f(t) \operatorname{div} Q_{n} v_{j} d x d t .
\end{aligned}
$$

Therefore letting $j \geq 1$ be fixed and $n \rightarrow \infty$, using the previous convergence together with the fact that $\theta_{0 n}^{\nu} \rightarrow \theta_{0}^{\nu}$ strongly in $L^{2}(D)$, we obtain for all $j \geq 1$

$$
\begin{gathered}
-\int_{D_{T}} f^{\prime}(t) \theta_{\nu} v_{j} d x d t-f(0) \int_{D} \theta_{0}^{\nu} v_{j} d x-\int_{D_{T}} f(t) \theta_{\nu} U_{\nu} \cdot \nabla v_{j} d x d t+ \\
\nu \int_{D_{T}} f(t) \Lambda_{\nu} \cdot \nabla v_{j} d x d t=-\int_{D_{T}} f(t) \operatorname{div} Q_{\nu} v_{j} d x d t
\end{gathered}
$$


Henceforth it holds for all $a \in W^{1,4}(D)$ and $f \in \mathcal{C}^{1}([0, T])$ such that $f(T)=0$ the following identity

$$
\begin{gathered}
-\int_{D_{T}} f^{\prime}(t) \theta_{\nu} a d x d t-f(0) \int_{D} \theta_{0}^{\nu} a d x-\int_{D_{T}} f(t) \theta_{\nu} U_{\nu} \cdot \nabla a d x d t \\
+\nu \int_{D_{T}} f(t) \Lambda_{\nu} \cdot \nabla a d x d t=-\int_{D_{T}} f(t) \operatorname{div} Q_{\nu} a d x d t
\end{gathered}
$$

In particular for $f \in \mathcal{D}(] 0, T[)$, we obtain (91) which is satisfied in $\mathcal{D}^{\prime}(] 0, T[)$. Thus we get in $\mathcal{D} \prime(D)$ and p.p. $t \in(0, T)$

$$
\partial_{t} \theta_{\nu}-B\left(U_{\nu}, \theta_{\nu}\right)+\nu \Lambda_{\nu}=-\operatorname{div} Q_{\nu}
$$

and we deduce that $\partial_{t} \theta_{\nu} \in L^{\frac{4}{3}}\left(0, T ;\left(W^{1,4}(D)\right)^{\prime}\right)$. Since $\theta_{\nu} \in L^{4}\left(0, T ; W^{1,4}(D)\right)$ then $\theta_{\nu} \in \mathcal{C}\left([0, T] ; L^{4}(D)\right)$ so that $\theta_{\nu}(0)$ is well defined and multiplying equation (91) by a function $f \in \mathcal{C}^{1}([0, T])$ such that $f(T)=0$ and integrating by parts, we obtain the equation

$$
\begin{gathered}
-\int_{D_{T}} f^{\prime}(t) \theta_{\nu} a d x d t-f(0) \int_{D} \theta_{\nu}(0) a d x-\int_{D_{T}} f(t) \theta_{\nu} U_{\nu} \cdot \nabla a d x d t \\
+\nu \int_{D_{T}} f(t) \Lambda_{\nu} \cdot \nabla a d x d t=-\int_{D_{T}} f(t) \operatorname{div} Q_{\nu} a d x d t
\end{gathered}
$$

for all $a \in W^{1,4}(D)$, thus comparing (98) and (100) we deduce that

$$
\theta_{\nu}(0)=\theta_{0}^{\nu}
$$

Next, since $\left(\theta_{n}(T)\right)_{n}$ is bounded in $L^{4}(D)$, then at least for a subsequence, $\left(\theta_{n}(T)\right)_{n}$ converge weakly in $L^{4}(D)$. Proceeding as previously using test functions $f \in \mathcal{C}^{1}([0, T])$ (with possibly the condition $f(0)=0$ for simplicity), we prove that

\section{Lemma 5}

$$
\theta_{n}(T) \rightarrow \theta_{\nu}(T) \text { weakly in } L^{4}(D) .
$$

It remains to prove the

Lemma 6 Let $\Lambda_{\nu}$ be the weak limit of $\left(\left|\nabla \theta_{n}\right|^{2} \nabla \theta_{n}\right)$ in $\mathbb{L}^{4 / 3}\left(D_{T}\right)$. We have

$$
\Lambda_{\nu}=\left|\nabla \theta_{\nu}\right|^{2} \nabla \theta_{\nu}
$$

Proof. We use the monotonicity of the operator $A$ defined in (92). We set

$$
A_{n}(\varphi)=\int_{0}^{T}\left\langle A\left(\theta_{n}\right)-A(\varphi), \theta_{n}-\varphi\right\rangle d t \geq 0, \quad \forall \varphi \in L^{4}\left(0, T ; W^{1,4}(D)\right) .
$$

We multiply equation (57) by $a_{j}$ and add these equations for $1 \leq j \leq n$, then integrating between 0 and $T$, we obtain

$$
\frac{1}{2}\left\|\theta_{n}(T)\right\|^{2}+\nu \int_{0}^{T}\left\|\nabla \theta_{n}\right\|_{L^{4}(D)}^{4} d s=\frac{1}{2}\left\|\theta_{0 n}^{\nu}\right\|^{2}-\int_{D_{T}} \operatorname{div} Q_{n} \theta_{n} d x d t
$$


We write

$$
\begin{gathered}
A_{n}(\varphi)=-\int_{D_{T}}\left|\nabla \theta_{n}\right|^{2} \nabla \theta_{n} \cdot \nabla \varphi d x d t-\int_{0}^{T}\left\langle A(\varphi), \theta_{n}-\varphi\right\rangle d t \\
+\frac{1}{\nu}\left[\frac{1}{2}\left(\left\|\theta_{0 n}^{\nu}\right\|^{2}-\left\|\theta_{n}(T)\right\|^{2} d x\right)-\int_{D_{T}} \operatorname{div} Q_{n} \theta_{n} d x d t\right] .
\end{gathered}
$$

Therefore by the convergence results given before, we get

$$
\begin{gathered}
\lim \sup A_{n}(\varphi) \leq-\int_{D_{T}} \Lambda_{\nu} \cdot \nabla \varphi d x d t-\int_{0}^{T}\left\langle A(\varphi), \theta_{\nu}-\varphi\right\rangle d t \\
+\frac{1}{\nu}\left[\frac{1}{2}\left(\left\|\theta_{0}^{\nu}\right\|^{2}-\left\|\theta_{\nu}(T)\right\|^{2} d x\right)-\int_{D_{T}} \operatorname{div} Q_{\nu} \theta_{\nu} d x d t\right] .
\end{gathered}
$$

Taking $a=\theta_{\nu}$ in equation (91) and integrating with respect to the time variable, we get

$$
\frac{1}{2}\left(\left\|\theta_{\nu}(T)\right\|^{2}-\left\|\theta_{0}^{\nu}\right\|^{2}\right)+\nu \int_{D_{T}} \Lambda_{\nu} \cdot \nabla \theta_{\nu} d x d t=-\int_{D_{T}} \operatorname{div} Q_{\nu} \theta_{\nu} d x d t .
$$

Coming back to inequality (103), we deduce that

$$
\limsup A_{n}(\varphi) \leq \int_{D_{T}} \Lambda_{\nu} \cdot\left(\nabla \theta_{\nu}-\nabla \varphi\right) d x d t-\int_{0}^{T}\left\langle A(\varphi), \theta_{\nu}-\varphi\right\rangle d t
$$

Therefore, for all $\varphi \in L^{4}\left(0, T ; W^{1,4}(D)\right)$ we have

$$
0 \leq \int_{D_{T}} \Lambda_{\nu} \cdot\left(\nabla \theta_{\nu}-\nabla \varphi\right) d x d t-\int_{0}^{T}\left\langle A(\varphi), \theta_{\nu}-\varphi\right\rangle d t
$$

Taking $\varphi=\theta_{\nu}-\lambda \psi$ with $\lambda>0$ and $\psi \in L^{4}\left(0, T ; W^{1,4}(D)\right)$, we get

$$
0 \leq \int_{D_{T}} \Lambda_{\nu} \cdot \nabla \psi d x d t-\int_{0}^{T}\left\langle A\left(\theta_{\nu}-\lambda \psi\right), \psi\right\rangle d t
$$

and since operator $A$ is hemicontinuous, then letting $\lambda \rightarrow 0$, we obtain

$$
0 \leq \int_{D_{T}} \Lambda_{\nu} \cdot \nabla \psi d x d t-\int_{D_{T}}\left|\nabla \theta_{\nu}\right|^{2} \nabla \theta_{\nu} \cdot \nabla \psi d x d t
$$

for all $\psi \in L^{4}\left(0, T ; W^{1,4}(D)\right)$ which leads to the result.

Going along the same lines, one can pass to the limit in the other equations thanks to the strong convergence results of Lemma 4 . One deduces that $U_{\nu}, M_{\nu}$ and $Q_{\nu}$ satisfy the weak formulations given in Theorem 2. To verify the corresponding initial conditions, one can proceed as for $\theta_{\nu}$. In order to prove energy estimates (65) and (73), we multiply (46) and (48) by a test function $f \in \mathcal{D}(] 0, T[)$ such that $f \geq 0$ and integrate between 0 and $T$. Thus using Lemma 2, we can take the lim inf which leads to the desired results. Hence we get a global weak solution with finite energy to problem $\left(\mathcal{P}_{\nu}\right)$. This ends proof of Theorem 2 . 


\section{Proof of Theorem 1}

\subsection{An auxiliary problem}

For $\nu>0$, let $\left(U_{\nu}, M_{\nu}, H_{\nu}, \theta_{\nu}, Q_{\nu}\right)$ be the solution of problem $\left(\mathcal{P}_{\nu}\right)$ provided by Theorem 2. In order to get some compacity result to deal with the limit of the nonlinear terms of the problem $\left(\mathcal{P}_{\nu}\right)$ when $\nu \rightarrow 0$, we introduce the following auxiliary function

$$
\zeta_{\nu}=\tau \gamma \operatorname{div} Q_{\nu}-\mathcal{K}\left(\theta_{\nu}\right)
$$

Taking the divergence of the heat-flux equation in (45), we easily see that $\zeta_{\nu}$ satisfies the equation

$$
\partial_{t} \zeta_{\nu}-\gamma \Delta \zeta_{\nu}=-\gamma \operatorname{div} Q_{\nu}-\frac{\tau \gamma}{2} \operatorname{div}\left(\operatorname{curl} U_{\nu} \times Q_{\nu}\right)-\partial_{t} \mathcal{K}\left(\theta_{\nu}\right)
$$

Multiplying the temperature equation in $(45)$ by $\mathcal{K}^{\prime}\left(\theta_{\nu}\right)$ we get

$$
\partial_{t} \mathcal{K}\left(\theta_{\nu}\right)+U_{\nu} \cdot \nabla \mathcal{K}\left(\theta_{\nu}\right)-\nu \mathcal{K}^{\prime}\left(\theta_{\nu}\right) \nabla \cdot\left(\left|\nabla \theta_{\nu}\right|^{2} \nabla \theta_{\nu}\right)=-\mathcal{K}^{\prime}\left(\theta_{\nu}\right) \operatorname{div} Q_{\nu}
$$

Hence $\zeta_{\nu}$ satisfies the auxiliary problem

$$
\begin{aligned}
& \partial_{t} \zeta_{\nu}-\gamma \Delta \zeta_{\nu}=g_{\nu}+\operatorname{div} G_{\nu}+\mu_{\nu} \text { in } D_{T} \\
& \zeta_{\nu}(0)=\zeta_{0}^{\nu} \text { in } D, \zeta_{\nu}=0 \text { on } \Gamma_{T}
\end{aligned}
$$

where $\zeta_{0}^{\nu}=\tau \gamma \operatorname{div} Q_{0}-\mathcal{K}\left(\theta_{0}^{\nu}\right)$ and

$$
\begin{gathered}
g_{\nu}=(\kappa-\gamma) \operatorname{div} Q_{\nu} \\
G_{\nu}=U_{\nu} \mathcal{K}\left(\theta_{\nu}\right)-\nu \kappa\left|\nabla \theta_{\nu}\right|^{2} \nabla \theta_{\nu}-3 \alpha \nu \theta_{\nu}^{2}\left|\nabla \theta_{\nu}\right|^{2} \nabla \theta_{\nu}-\frac{\tau \gamma}{2} \operatorname{curl} U_{\nu} \times Q_{\nu} \\
\mu_{\nu}=6 \nu \alpha \theta_{\nu}\left|\nabla \theta_{\nu}\right|^{4}+3 \alpha \theta_{\nu}^{2} \operatorname{div} Q_{\nu}
\end{gathered}
$$

We note that $\zeta_{0}^{\nu}$ converge strongly in $L^{\frac{4}{3}}(D)$ towards $\zeta_{0}=\tau \gamma \operatorname{div} Q_{0}-\mathcal{K}\left(\theta_{0}\right)$ and we have the following results

Lemma 7 There exists $C>0$ which is independent of $\nu$ such that

$$
\begin{aligned}
& \left\|g_{\nu}\right\|_{L^{2}\left(0, T ; L^{2}(D)\right)} \leq C \\
& \left\|G_{\nu}\right\|_{L^{4 / 3}\left(0, T ; \mathbb{L}^{12 / 11}(D)\right)} \leq C \\
& \left\|\mu_{\nu}\right\|_{L^{1}\left(0, T ; L^{1}(D)\right)} \leq C
\end{aligned}
$$

Proof. We use the energy estimates (46) and (48) satisfied by $\left(U_{\nu}, M_{\nu}, H_{\nu}, \theta_{\nu}, Q_{\nu}\right)$. So clearly $g_{\nu}$ is bounded in $L^{2}\left(0, T ; L^{2}(D)\right)$. We write $G_{\nu}$ in the form $G_{\nu}=G_{1, \nu}-$ $G_{2, \nu}-G_{3, \nu}-G_{4, \nu}$ with

$$
\begin{gathered}
G_{1, \nu}=U_{\nu} \mathcal{K}\left(\theta_{\nu}\right), G_{2, \nu}=\nu \kappa\left|\nabla \theta_{\nu}\right|^{2} \nabla \theta_{\nu} \\
G_{3, \nu}=3 \alpha \nu \theta_{\nu}^{2}\left|\nabla \theta_{\nu}\right|^{2} \nabla \theta_{\nu}, G_{4, \nu}=\frac{\tau \gamma}{2} \operatorname{curl} U_{\nu} \times Q_{\nu} .
\end{gathered}
$$

Since $L^{2}(0, T ; \mathcal{U})$ is continuously embedded in $L^{2}\left(0, T ; \mathbb{L}^{6}(D)\right)$ then we see that $U_{\nu}$ is bounded in $L^{2}\left(0, T ; \mathbb{L}^{6}(D)\right)$ with respect to $\nu$ and as $\mathcal{K}\left(\theta_{\nu}\right)$ is bounded in $L^{\infty}\left(0, T ; L^{\frac{4}{3}}(D)\right)$, 
we deduce that $G_{1, \nu}$ is bounded in $L^{2}\left(0, T ; \mathbb{L}^{12 / 11}(D)\right) . \quad G_{2, \nu}$ is clearly bounded in $L^{4 / 3}\left(0, T ; \mathbb{L}^{4 / 3}(D)\right)$. Next since $Q_{\nu}$ is bounded in $L^{\infty}\left(0, T ; \mathbb{L}^{2}(D)\right) \cap L^{2}\left(0, T ; \mathbb{H}^{1}(D)\right)$ then using an interpolation result we get that $Q_{\nu}$ is bounded in $L^{4}\left(0, T ; \mathbb{L}^{3}(D)\right)$. Therefore as $\operatorname{curl} U_{\nu}$ is bounded in $L^{2}\left(0, T ; \mathbb{L}^{2}(D)\right)$, we conclude that $G_{4, \nu}$ is bounded in $L^{4 / 3}\left(0, T ; \mathbb{L}^{6 / 5}(D)\right)$. Now we write $\left|G_{3, \nu}\right|=(3 \alpha \nu) h_{\nu} k_{\nu}$ with

$$
\begin{gathered}
h_{\nu}=\left|\theta_{\nu}\right|^{3 / 2}\left|\nabla \theta_{\nu}\right|^{3} \in L^{4 / 3}\left(0, T ; L^{4 / 3}(D)\right) \\
k_{\nu}=\left|\theta_{\nu}\right|^{1 / 2} \in L^{\infty}\left(0, T ; L^{8}(D)\right) .
\end{gathered}
$$

So $G_{3, \nu} \in L^{4 / 3}\left(0, T ; L^{8 / 7}(D)\right)$ and p.p. $t \in(0, T)$

$$
\begin{gathered}
\left\|G_{3, \nu}(t)\right\|_{L^{8 / 7}(D)} \leq 3 \alpha \nu\left\|h_{\nu}(t)\right\|_{L^{4 / 3}(D)}\left\|k_{\nu}(t)\right\|_{L^{8}(D)} \\
\leq 3 \alpha \nu\left(\int_{D}\left|\theta_{\nu}\right|^{2}\left|\nabla \theta_{\nu}\right|^{4} d x\right)^{3 / 4}\left\|\theta_{\nu}\right\|_{L^{4}(D)}^{1 / 2}
\end{gathered}
$$

then

$$
\left\|G_{3, \nu}\right\|_{L^{4 / 3}\left(0, T ; L^{8 / 7}(D)\right)} \leq 3 \alpha \nu\left(\int_{D_{T}}\left|\theta_{\nu}\right|^{2}\left|\nabla \theta_{\nu}\right|^{4} d x d t\right)^{3 / 4}\left\|\theta_{\nu}\right\|_{L^{\infty}\left(0, T ; L^{4}(D)\right)}^{1 / 2}
$$

therefore $G_{3, \nu}$ is uniformly bounded in $L^{4 / 3}\left(0, T ; \mathbb{L}^{8 / 7}(D)\right)$ with respect to $\nu$ which implies that $G_{\nu}$ is bounded in $L^{4 / 3}\left(0, T ; \mathbb{L}^{12 / 11}(D)\right)$. To deal with $\mu_{\nu}$, we split it into two terms $\mu_{\nu}=\mu_{1, \nu}+\mu_{2, \nu}$ with $\mu_{1, \nu}=6 \nu \alpha \theta_{\nu}\left|\nabla \theta_{\nu}\right|^{4}$ and $\mu_{2, \nu}=3 \alpha \theta_{\nu}^{2} \operatorname{div} Q_{\nu}$. Then clearly the second term $\mu_{2, \nu}$ is bounded in $L^{2}\left(0, T ; L^{1}(D)\right)$ whereas for the first term, we have

$$
\begin{aligned}
& \int_{D_{T}}\left|\mu_{1, \nu}\right| d x d t=6 \alpha \nu \int_{D_{T}}\left|\theta_{\nu}\right|\left|\nabla \theta_{\nu}\right|^{4} d x d t \leq \\
& 6 \alpha \nu \int_{\left\{\left|\theta_{\nu}\right| \leq 1\right\}}\left|\nabla \theta_{\nu}\right|^{4}+6 \alpha \nu \int_{\left\{\left|\theta_{\nu}\right|>1\right\}}\left|\theta_{\nu}\right|^{2}\left|\nabla \theta_{\nu}\right|^{4} d x d t \leq C
\end{aligned}
$$

The Lemma is then proved.

To use the known regularity results on solutions of parabolic equations, we split the function $\zeta_{\nu}$ into two terms $\zeta_{1, \nu}$ and $\zeta_{2, \nu}$ where $\zeta_{1, \nu}$ satisfies the problem

$$
\begin{gathered}
\partial_{t} \zeta_{1, \nu}-\gamma \Delta \zeta_{1, \nu}=g_{\nu}+\operatorname{div} G_{\nu} \text { in } D_{T} \\
\zeta_{1, \nu}(0)=\zeta_{0}^{\nu} \text { in } D, \zeta_{1, \nu}=0 \text { on } \Gamma_{T}
\end{gathered}
$$

with $g_{\nu}$ and $G_{\nu}$ bounded in $L^{12 / 11}\left(0, T ; \mathbb{L}^{12 / 11}(D)\right), \zeta_{0}^{\nu}$ bounded in $L^{\frac{4}{3}}(D)$ whereas the function $\zeta_{2, \nu}$ verifies the problem

$$
\begin{aligned}
& \partial_{t} \zeta_{2, \nu}-\gamma \Delta \zeta_{2, \nu}=\mu_{\nu} \text { in } D_{T} \\
& \zeta_{2, \nu}(0)=0 \text { in } D, \zeta_{2, \nu}=0 \text { on } \Gamma_{T}
\end{aligned}
$$

with $\mu_{\nu}$ bounded in $L^{1}\left(0, T ; L^{1}(D)\right)$. We have the result

Lemma 8 There exists $C>0$ which is independent of $\nu$ such that

$$
\begin{aligned}
& \left\|\zeta_{1, \nu}\right\|_{L^{12 / 11}\left(0, T ; W^{1,12 / 11}(D)\right)}+\left\|\partial_{t} \zeta_{1, \nu}\right\|_{L^{12 / 11}\left(0, T ; W^{-1,12 / 11}(D)\right)} \leq C \\
& \left\|\zeta_{2, \nu}\right\|_{L^{p}\left(0, T ; W^{1, p}(D)\right)}+\left\|\partial_{t} \zeta_{2, \nu}\right\|_{L^{p}\left(0, T ; W^{-1, p}(D)\right)+L^{1}\left(0, T ; L^{1}(D)\right)} \leq C
\end{aligned}
$$

for all $1 \leq p<\frac{5}{4}$. 
Proof. The bounds for $\zeta_{1, \nu}$ result from the classical $L^{p}$ estimates of solutions of parabolic equations (see for example [9], [12] and the references therein) whereas for $\zeta_{2, \nu}$, we use a result given in [2], see also [4]. The estimate of $\partial_{t} \zeta_{2, \nu}$ follows from the equation (116).

Combining the above estimates and using Aubin's compactness lemma we get the convergence results

Lemma 9 For subsequences we have

$$
\begin{aligned}
& \zeta_{1, \nu} \rightarrow \zeta_{1} \text { strongly in } L^{12 / 11}\left(0, T ; L^{q_{1}}(D)\right), 1 \leq q_{1}<(12 / 11)^{\star}=12 / 7 \\
& \zeta_{2, \nu} \rightarrow \zeta_{2} \text { strongly in } L^{p}\left(0, T ; L^{q_{2}}(D)\right), 1 \leq q_{2}<p^{\star}=\frac{3 p}{3-p}, \quad 1 \leq p<5 / 4 \\
& \zeta_{\nu} \rightarrow \zeta \text { strongly in } L^{12 / 11}\left(0, T ; L^{q_{1}}(D)\right), 1 \leq q_{1}<12 / 7 .
\end{aligned}
$$

The strong convergence of $\zeta_{\nu}$ is crucial to obtain the limit in the nonlinear term $\mathcal{K}\left(\theta_{\nu}\right)$ since the regularized equation of $\theta_{\nu}$ does not provide any uniform bound of the space derivative. We will precise this point in the following subsection.

\subsection{Convergence in the temperature equation as $\nu \rightarrow 0$}

Let $\nu>0$ and $\left(U_{\nu}, M_{\nu}, H_{\nu}, \theta_{\nu}, Q_{\nu}\right)$ be the solution of problem $\left(\mathcal{P}_{\nu}\right)$ provided by Theorem 2. From the estimates (46), we deduce that

Lemma 10 For a subsequence, we have

$$
\begin{gathered}
\theta_{\nu} \rightarrow \theta \text { weakly }-* \text { in } L^{\infty}\left(0, T ; L^{4}(D)\right) \\
\nu\left|\nabla \theta_{\nu}\right|^{2} \nabla \theta_{\nu} \rightarrow 0 \text { strongly in } \mathbb{L}^{4 / 3}\left(D_{T}\right) .
\end{gathered}
$$

Next, assume momentarily that

$$
\begin{gathered}
U_{\nu} \rightarrow U \text { strongly in } \mathbb{L}^{2}\left(0, T ; \mathcal{U}_{0}\right) \\
\operatorname{div} Q_{\nu} \rightarrow \operatorname{div} Q \text { weakly in } L^{2}\left(D_{T}\right)
\end{gathered}
$$

then we can perform the limit when $\nu \rightarrow 0$ in the equation of $\theta_{\nu}$. We get the result

Lemma $11 \theta \in L^{\infty}\left(0, T ; L^{4}(D)\right)$ is a weak solution of the transport equation

$$
\begin{aligned}
& \partial_{t} \theta+U \cdot \nabla \theta=-\operatorname{div} Q \text { in } D_{T} \\
& \theta(0)=\theta_{0} \text { in } D .
\end{aligned}
$$

Moreover, $\theta \in C\left([0, T] ; L^{q}(D)\right)$ for all $q<4$ and $\theta \in C\left([0, T] ; L^{4}(D)\right.$ weak $)$.

We recall that $\theta \in L^{\infty}\left(0, T ; L^{4}(D)\right)$ is a weak solution of (121) if

$$
\int_{D_{T}} \theta\left(\partial_{t} a+U \cdot \nabla a\right) d x d t=\int_{D_{T}} \operatorname{div} Q a d x d t-\int_{D} \theta_{0} a(0) d x
$$

for all $a \in \mathcal{D}\left(\left[0, T[\times \bar{D})\right.\right.$ so equation (121) is satisfied in $\mathcal{D}^{\prime}\left(D_{T}\right)$. The regularity result given in Lemma 11 is well known in transport equation theory as said in Remark 2.3, which gives a sense to the initial condition in (121). Moreover, we have 


\section{Lemma 12}

$$
\theta_{\nu}(T) \rightarrow \theta(T) \text { weakly in } L^{2}(D)
$$

Proof. $\theta(T)$ is well defined in $L^{2}(D)$, let $v$ be the weak limit of $\theta_{\nu}(T)$ in $L^{4}(D)$ and $L^{2}(D)$. We use test functions $a \in \mathcal{D}(D)$ in the equation (52) of $\theta_{\nu}$, we multiply by $f \in \mathcal{D}(] 0, T])$ and integrating between 0 and $T$, we get

$$
\begin{gathered}
f(T) \int_{D} \theta_{\nu}(T) a d x=\int_{D_{T}} f(t) \theta_{\nu} U_{\nu} \cdot \nabla a d x d t \\
-\nu \int_{D_{T}} f(t)\left|\nabla \theta_{\nu}\right|^{2} \nabla \theta_{\nu} \cdot \nabla a d x d t-\int_{D_{T}} f(t) \operatorname{div} Q_{\nu} a d x d t
\end{gathered}
$$

thus passing to the limit we get

$$
f(T) \int_{D} v a d x=\int_{D_{T}} f(t) \theta U \cdot \nabla a d x d t-\int_{D_{T}} f(t) \operatorname{div} Q a d x d t .
$$

Using equation (121) of $\theta$, we deduce that for all $a \in \mathcal{D}(D), f \in \mathcal{D}(] 0, T])$

$$
f(T) \int_{D} v a d x=f(T) \int_{D} \theta(T) a d x
$$

hence $v=\theta(T)$.

Consider now the equation of $\theta_{\nu}$ from (45) that we rewrite using $\zeta_{\nu}$ in the form

$$
\begin{aligned}
& \partial_{t} \theta_{\nu}+U_{\nu} \cdot \nabla \theta_{\nu}-\nu \nabla \cdot\left(\left|\nabla \theta_{\nu}\right|^{2} \nabla \theta_{\nu}\right)+\frac{1}{\tau \gamma} \mathcal{K}\left(\theta_{\nu}\right)=-\frac{1}{\tau \gamma} \zeta_{\nu} \text { in } D_{T} \\
& \theta_{\nu}(0)=\theta_{0}^{\nu} \text { in } D, \quad \nu\left|\nabla \theta_{\nu}\right|^{2} \nabla \theta_{\nu} \cdot \mathbf{n}=0 \text { on } \Gamma_{T} .
\end{aligned}
$$

Under the hypothesis (120), we have

Lemma 13 Let $\chi$ be the weak-* limit of $\mathcal{K}\left(\theta_{\nu}\right)$ in $L^{\infty}\left(0, T ; L^{4 / 3}(D)\right)$. We have

$$
\chi=\mathcal{K}(\theta) .
$$

Proof. We use the monotonicity of the function $\mathcal{K}$ and the strong convergence in $L^{1}\left(0, T ; L^{4 / 3}(D)\right)$ of $\zeta_{\nu}$ given in Lemma 9. We set

$$
a_{\nu}(\varphi)=\int_{D_{T}}\left(\mathcal{K}\left(\theta_{\nu}\right)-\mathcal{K}(\varphi)\right)\left(\theta_{\nu}-\varphi\right) d x d t \geq 0, \quad \forall \varphi \in L^{\infty}\left(0, T ; L^{4}(D)\right) .
$$

We multiply equation (123) by $\theta_{\nu}$ and integrate over $D_{T}$ to get

$$
\begin{gathered}
\frac{1}{\tau \gamma} \int_{D_{T}} \mathcal{K}\left(\theta_{\nu}\right) \theta_{\nu} d x d t=-\frac{1}{\tau \gamma} \int_{D_{T}} \zeta_{\nu} \theta_{\nu} d x d t-\nu \int_{D_{T}}\left|\nabla \theta_{\nu}\right|^{4} d x d t \\
-\frac{1}{2} \int_{D} \theta_{\nu}^{2}(T) d x+\frac{1}{2} \int_{D}\left(\theta_{0}^{\nu}\right)^{2} d x
\end{gathered}
$$

Then

$$
\begin{aligned}
a_{\nu}(\varphi) & =-\int_{D_{T}} \mathcal{K}\left(\theta_{\nu}\right) \varphi d x d t-\int_{D_{T}} \mathcal{K}(\varphi)\left(\theta_{\nu}-\varphi\right) d x d t-\int_{D_{T}} \zeta_{\nu} \theta_{\nu} d x d t \\
& -\tau \gamma \nu \int_{D_{T}}\left|\nabla \theta_{\nu}\right|^{4} d x d t-\frac{\tau \gamma}{2} \int_{D} \theta_{\nu}^{2}(T) d x+\frac{\tau \gamma}{2} \int_{D}\left(\theta_{0}^{\nu}\right)^{2} d x
\end{aligned}
$$


$>$ From Lemma 12, we have

$$
\liminf \int_{D}\left|\theta_{\nu}(T)\right|^{2} d x \geq \int_{D}|\theta(T)|^{2} d x
$$

therefore using hypothesis (44) we obtain

$$
\begin{array}{r}
\lim \sup a_{\nu}(\varphi) \leq-\int_{D_{T}} \chi \varphi d x d t-\int_{D_{T}} \mathcal{K}(\varphi)(\theta-\varphi) d x d t-\int_{D_{T}} \zeta \theta d x d t \\
-\frac{\tau \gamma}{2} \int_{D} \theta^{2}(T) d x+\frac{\tau \gamma}{2} \int_{D} \theta_{0}^{2} d x
\end{array}
$$

for all $\varphi$ in $L^{\infty}\left(0, T ; L^{4}(D)\right)$. On the other hand, taking the limit in the weak formulation of problem (123), we deduce that $\theta$ satisfies the equation

$$
\begin{aligned}
& \partial_{t} \theta+U \cdot \nabla \theta+\frac{1}{\tau \gamma} \chi=-\frac{1}{\tau \gamma} \zeta \text { in } D_{T} \\
& \theta(0)=\theta_{0} \text { in } D .
\end{aligned}
$$

Multiplying the equation by $\theta$ we get

$$
-\int_{D_{T}} \zeta \theta d x d-\frac{\tau \gamma}{2} \int_{D} \theta^{2}(T) d x+\frac{\tau \gamma}{2} \int_{D} \theta_{0}^{2} d x=\int_{D_{T}} \chi \theta d x d t .
$$

Therefore

$$
\lim \sup a_{\nu}(\varphi) \leq \int_{D_{T}} \chi(\theta-\varphi) d x d t-\int_{D_{T}} \mathcal{K}(\varphi)(\theta-\varphi) d x d t
$$

We deduce that

$$
\int_{D_{T}}(\chi-\mathcal{K}(\varphi))(\theta-\varphi) d x d t \geq 0, \quad \forall \varphi \in L^{\infty}\left(0, T ; L^{4}(D)\right) .
$$

So, taking in (125) $\varphi=\theta-\lambda \psi$ with $\lambda>0, \psi \in L^{\infty}\left(0, T ; L^{4}(D)\right)$, we get

$$
\int_{D_{T}}(\chi-\mathcal{K}(\theta-\lambda \psi)) \psi d x d t \geq 0, \quad \forall \psi \in L^{\infty}\left(0, T ; L^{4}(D)\right), \forall \lambda>0
$$

and letting $\lambda \rightarrow 0$, we obtain

$$
\int_{D_{T}}(\chi-\mathcal{K}(\theta)) \psi d x d t \geq 0, \quad \forall \psi \in L^{\infty}\left(0, T ; L^{4}(D)\right)
$$

and we conclude that $\chi=\mathcal{K}(\theta)$.

\subsection{End of proof of theorem 1}

Let $\left(U_{\nu}, M_{\nu}, H_{\nu}, \theta_{\nu}, Q_{\nu}\right)$ be the solution of problem $\left(\mathcal{P}_{\nu}\right)$ provided by Theorem 2 for $\nu>0$. The energy estimates (46) and (48) lead to the following uniform bounds with respect to $\nu$

\section{Lemma 14}

- $\left(U_{\nu}\right)$ is uniformly bounded in $L^{\infty}\left(0, T ; \mathcal{U}_{0}\right) \cap L^{2}(0, T ; \mathcal{U})$ 
- $\left(M_{\nu}\right)$ and $\left(H_{\nu}\right)$ are uniformly bounded in $L^{\infty}\left(0, T ; \mathbb{L}^{2}(D)\right) \cap L^{2}\left(0, T ; \mathbb{H}_{t}^{1}(D)\right)$

- $\left(M_{\nu} \times H_{\nu}\right)$ is uniformly bounded in $L^{2}\left(0, T ; \mathbb{L}^{2}(D)\right)$

- $\left(Q_{\nu}\right)$ is uniformly bounded in $L^{\infty}\left(0, T ; \mathbb{L}^{2}(D)\right) \cap L^{2}\left(0, T ; \mathbb{H}_{n}^{1}(D)\right)$

To deal with the nonlinear terms of the equations, we need to estimate the time derivatives $\left(\partial_{t} U_{\nu}\right),\left(\partial_{t} M_{\nu}\right)$ and $\left(\partial_{t} Q_{\nu}\right)$. For the first one, we deduce from the weak formulation of the equation of $U_{\nu}$ that in $\mathcal{D}^{\prime}(D)$, p.p. $t \in(0, T)$ it holds

$$
\partial_{t} U_{\nu}=-\mathcal{L}_{\nu}
$$

where the linear form $\mathcal{L}_{\nu}$ is defined on $\mathcal{U}$ by

$$
\begin{aligned}
\left\langle\mathcal{L}_{\nu}, \varphi\right\rangle & =\int_{D}\left(U_{\nu} \cdot \nabla\right) U_{\nu} \cdot \varphi d x+\eta \int_{D} \nabla U_{\nu} \cdot \nabla \varphi d x+\int_{D} \rho\left(\theta_{\nu}\right) g \cdot \varphi d x \\
& -\mu_{0} \int_{D}\left(M_{\nu} \cdot \nabla\right) H_{\nu} \cdot \varphi d x-\frac{\mu_{0}}{2} \int_{D} M_{\nu} \times H_{\nu} \cdot \operatorname{curl} \varphi d x
\end{aligned}
$$

Therefore $\mathcal{L}_{\nu} \in \mathcal{U}^{\prime}$ p.p. $t \in(0, T)$ and

$$
\begin{gathered}
\left\|\mathcal{L}_{\nu}\right\|_{\mathcal{U}^{\prime}} \leq C\left(\left\|U_{\nu}\right\|_{H^{1}(D)}^{2}+\left\|U_{\nu}\right\|_{H^{1}(D)}+\left\|\rho\left(\theta_{\nu}\right)\right\|+\right. \\
\left.\left\|M_{\nu}\right\|_{H^{1}(D)}\left\|H_{\nu}\right\|_{H^{1}(D)}+\left\|M_{\nu} \times H_{\nu}\right\|\right) \leq C
\end{gathered}
$$

thanks to the bounds of Lemma 14. We deduce that $\partial_{t} U_{\nu}$ is uniformly bounded in $L^{1}\left(0, T ; \mathcal{U}^{\prime}\right)$. and since the same proofs work for $\partial_{t} M_{\nu}$ and $\partial_{t} Q_{\nu}$, we get

Lemma $15\left(\partial_{t} U_{\nu}\right),\left(\partial_{t} M_{\nu}\right)$ and $\left(\partial_{t} Q_{\nu}\right)$ are uniformly bounded with respect to $\nu$ in $L^{1}\left(0, T ; \mathcal{U}^{\prime}\right), L^{1}\left(0, T ;\left(H_{t}^{1}(D)\right)^{\prime}\right)$ and $L^{1}\left(0, T ;\left(H_{n}^{1}(D)\right)^{\prime}\right)$ respectively.

Hence we get the convergence results

Lemma 16 There exists subsequences still denoted $\left(U_{\nu}\right),\left(M_{\nu}\right),\left(H_{\nu}\right),\left(Q_{\nu}\right)$ such that when $\nu \rightarrow 0$

$$
\begin{aligned}
& U_{\nu} \rightarrow U \text { weakly }-\star \text { in } L^{\infty}\left(0, T ; \mathcal{U}_{0}\right) \text { and weakly in } L^{2}(0, T ; \mathcal{U}) \\
& M_{\nu} \rightarrow M \text { weakly }-\star \text { in } L^{\infty}\left(0, T ; \mathbb{L}^{2}(D)\right) \text { and weakly in } L^{2}\left(0, T ; \mathbb{H}_{t}^{1}(D)\right) \\
& H_{\nu} \rightarrow H \text { weakly }-\star \text { in } L^{\infty}\left(0, T ; \mathbb{L}^{2}(D)\right) \text { and weakly in } L^{2}\left(0, T ; \mathbb{H}_{t}^{1}(D)\right) \\
& Q_{\nu} \rightarrow Q \text { weakly }-\star \text { in } L^{\infty}\left(0, T ; \mathbb{L}^{2}(D)\right) \text { and weakly in } L^{2}\left(0, T ; \mathbb{H}_{n}^{1}(D)\right)
\end{aligned}
$$

and the following strong convergence results hold

$$
\left(U_{\nu}, M_{\nu}, H_{\nu}, Q_{\nu}\right) \rightarrow(U, M, H, Q) \text { strongly in }\left(\mathbb{L}^{2}\left(D_{T}\right)\right)^{4}
$$

where $H=\mathcal{H}(M, F)$.

Once again, the strong convergence of $\left(H_{\nu}\right)$ is a consequence of subsection 1.3.

Notice that from Lemma 16, the hypotheses (120) are fulfilled leading to the convergence of the temperature of the previous subsection. Proceeding as for the proof of Theorem 
2, we can verify that $(U, M, H, \theta, Q)$ is a weak solution with finite energy of problem $(\mathcal{P})$ which leads to the conclusion of the first part of Theorem 1.

Let us prove the second part of the Theorem, which is the $L^{p}-L^{q}$ - regularity for the temperature $\theta$. In this purpose, it is enough to show that

Lemma 17 Under hypotheses (36), the regularized temperature $\theta_{\nu}$ is uniformly bounded in $L^{36 / 11}\left(0, T ; L^{36 / 7}(D)\right)$ with respect to $\nu$.

Proof. From (117) and using the Sobolev embedding $W^{1,12 / 11}(D) \subset L^{12 / 7}(D)$, we see that $\left(\zeta_{\nu}\right)$ is uniformly bounded in $L^{12 / 11}\left(0, T ; L^{12 / 7}(D)\right)$ then from the relation $\alpha \theta_{\nu}^{3}=$ $-\zeta_{\nu}+\tau \gamma \operatorname{div} Q_{\nu}-\kappa \theta_{\nu}$, we deduce that $\left(\theta_{\nu}\right)$ is uniformly bounded in $L^{36 / 11}\left(0, T ; L^{36 / 7}(D)\right)$.

\section{References}

[1] Y. Amirat, K. Hamdache, Global weak solutions to a ferrofluidflow model, Math. Methods Appl. Sci. 31(2), 123-151 (2007)

[2] Y. Amirat, K. Hamdache, Heat transfer in incompressible magnetic fluid, Journal of Mathematical Fluid Mechanics V. 14, N. 2, 217-247 (2012)

[3] I. Bihari, A generalization of a lemma of Bellman and its application to the uniqueness problems of differential equations, Acta Marthgematica Hungarica. Volume 7, Numéro 1, p-p 81-94 (1956)

[4] L. Boccardo, T. Gallouet, Nonlinear elliptic and parabolic equations involving measures data, Functionnal Analysis, 87 , 149-169 (1989)

[5] F. Boyer, A propos de l'équation de transport. Problèmes de traces. Régularité des solutions, Conférence Grenoble, 10 février 2005.

[6] M.C. Cattaneo Sur une forme de l'équation de la chaleur éleminant le paradoxe d'une propagation instannée, Note CRAS, Série I, 247, 431-433 (1958)

[7] R. Dautary, J.L Lions, Analyse mathématique et calcul numérique pour les sciences et les techniques, Tome 2, Masson (1985)

[8] R.J. Di Perna, P.L. Lions Ordinary differential equations, transport theory and Sobolev spaces, Invent. Math 511-547 (1989)

[9] H. Dong and D. Kim, $L_{p}$ solvability of divergence type parabolic and elliptic systems with partially BMO coefficients, Calc. Var. 40 : 357-389 (2011)

[10] E. Feireisl, Dynamics of viscous compressible fluids, Oxford Lecture Series in Mathematics and it Applications. 26

[11] E. Feireisl, J. Málek, On the Navier-Stokes equations with temperature-dependant transport coefficients, Differential Equations and Nonlinear Mechanics, 90616, 1-14 (2006) 
[12] E. Feireisl, A. Novotný Singular limits in theormodynamics of viscous fluids, Advances in Mathematical Fluids Mechanics. Birkhauser

[13] R.A Guyer, J.A Krumhansl Solution of the linearized phonon Boltzman equation, Physical Review, 148(2), 766-778 (1996)

[14] C. Lin, L.E. Payne, Continuous dependence on spatial geometry for the generalized Maxwell-Cattaneou system, Math. Meth. in the Appl. Sciences, 24, 1113-1124 (2001)

[15] C. Lin, L.E. Payne, Continuous dependence of heat flux on spatial geometry for generalized Maxwell-Cattaneo system, Z. angew. Math. Phys. Doi 10.1007/s00033004-1136-1 (2004)

[16] J.L. Lions, Quelques méthodes de résolution de problèmes aux limites non linéaires, Paris, Dunod (1969)

[17] L.E. Payne, P.W. Schaefer, J.C. Song, Improved bounds for some nonstandard problems in generalized heat conduction, J. Math. anal. Appl. 298, 325-340 (2004)

[18] L.E. Payne, J.C. Song, Improved spatial decay bounds in generalized heat conduction, Z. angew. Math. Phys. 56, 805-820 (2005)

[19] S. Pranesh, R.V. Kiran, Study of Raylaigh-Bénard magneto convection micro polar fluid with Maxwell-Cattaneo law, Applied mathematics, 3, 417-424 (2012)

[20] S. Pranesh, A. K. Narayanappa, effect of non-uniform concentration gradient on the Onset of double-diffusive convection in micro polar fluid, Applied mathematics, 470- 480 (2010)

[21] J. Simon, Compactness sets in the space $L^{p}(0, T ; B)$, Ann. Math. Pura. Appl. IV, Vol 146 (1985), pp. 65-96

[22] R.Temam, Navier-Stokes equations, Theory and numerical analysis, North Holland (1979)

[23] M.P. Vernotte Les paradoxes de la théorie continue de l'équation de la chaleur, C.R Acad. Sci. Paris 246, 3154 (1958) 